\title{
Joint Iterative Channel Estimation and Frequency-Domain Turbo Equalization for Single-Carrier Spatial Modulation
}

\author{
Yan Zhao, Yue Xiao, Member, IEEE, Ping Yang, Senior member, IEEE, Binhong Dong, Lajos \\ Hanzo, Fellow IEEE
}

\begin{abstract}
Single carrier frequency-domain turbo equalization (SC-FDTE) has gained widespread adoption in the emerging broadband spatial modulation (S$\mathrm{M})$ systems operating in frequency selective channels, where the channel model considered is a quasi-static Rayleigh fading channel. In this paper, a new class of robust FDTE designs based on the minimum meansquare error (MMSE) criterion is conceived for broadband single-carrier SM (SC-SM) systems relying on realistic imperfect channel knowledge. First, a robust time-domain soft-decision feedback (TDSDF) aided FDTE is proposed to cope with channel estimation errors at the receiver. Furthermore, its robust frequencydomain soft-decision feedback (FDSDF) aided counterpart is derived to offer a low-complexity approximate solution. Finally, by exploiting the carefully selected reliable soft-decision output of the channel decoder as pilots, we refine the resultant decision-directed channel estimation. As a benefit, the performance of the two robust FDTEs can be further improved. Both our simulation results and our extrinsic information transfer (EXIT) chart analysis demonstrate that the proposed robust FDTEs achieve significant performance improvements over the conventional FDTEs.
\end{abstract}

Index Terms - Single-carrier (SC), spatial modulation (SM), frequency-domain turbo equalizer (FDTE), imperfect channel knowledge.

\section{INTRODUCTION}

$\mathbf{S}$ PATIAL modulation (SM) has been widely considered as an attractive low-cost single radio-frequency $(\mathrm{RF})$ multiple-input multiple-output (MIMO) technique as a benefit of its simplified transceiver structure and low power dissipation [1]-[4]. Despite having multiple transmit antennas (TAs), in the original SM scheme only a single $\mathrm{TA}$ and a single RF-chain is activated for transmitting a classic modulated symbol at any time instant, hence extra information may be conveyed by the index of the activated TA in addition to the symbols of a conventional modulation scheme.

Y. Zhao, Y. Xiao, P. Yang and B. Dong are with the National Key Laboratory of Science and Technology on Communications, University of Electronic Science and Technology of China 611731 Sichuan, China. (e-mail: zyshitou@126.com, xiaoyue@uestc.edu.cn, yang.ping@uestc.edu.cn, bhdong@uestc.edu.cn).

L. Hanzo is with the School of Electronics and Computer Science, University of Southampton, Southampton SO17 1BJ, U.K. (e-mail: lh@ecs.soton.ac.uk).

The financial supports of the National Science Foundation of China under Grant number 61671131, National Key R\&D Program of China (No.2018YFC0807101), and of Sichuan Science and Technology Program (2018HH0138) are gratefully acknowledged. L. Hanzo would also like to thank the EPSRC for their financial support of the Grant $\mathrm{EP} / \mathrm{P} \emptyset 3 \mathrm{H} 2 \mathrm{FH} / 1$ and the $\mathrm{ERC}$ for his Advanced Fellow Grant QuantCom.
In the previous studies of SM, a flurry of research has been focused on the design of low-complexity transceivers for flat-fading channels [5]-[9], but there is a paucity of SM-contributions designed for frequency-selective fading channels. In order to support high-rate broadband SM transmission, single-carrier (SC) SM [10] and multicarrier SM orthogonal frequency division multiplexing (OFDM) [11] have been proposed for frequency-selective fading channels. Compared to SM-OFDM, SC-SM constitutes a more promising alternative for broadband SM scenarios, since it exhibits reduced peak-to-average power ratios, offers improved multi-path diversity gains and retains the single RF structure.

As a benefit of the attractive SC-SM features described above, hard-decision based equalizers have been proposed for SC-SM [12]-[15]. In order to achieve improved coding gains, turbo equalization [16]-[17] was integrated with the soft-input soft-output detectors of SC-SM [18]-[23], yielding either a frequency-domain turbo equalizer (FDTE) [18]-[20] or a time-domain turbo equalizer (TDTE) [21][23]. However, most of the above-mentioned detectors relied on the idealized simplifying assumption that perfect channel state information (CSI) is available at the receiver. Explicitly, practical channel estimation has not been considered in their equalization processes. In practice, it is quite challenging to acquire accurate CSI in multiple antenna aided systems. However, realistic imperfect channel estimation degrades the equalization performance [10], [24]-[27]. Specifically, the authors of [22] demonstrated that under imperfect channel estimation, the TDTE of [22] suffered from significant performance losses due to the inaccuracy of the estimated CSI. Afterwards, the authors of [23] embarked on improving the performance whilst reducing the complexity of the TDTE under the idealized simplifying assumption of having perfect CSI, whilst setting aside the realistic imperfect channel estimation scenario for future research. Explicitly, given that the impact of imperfect channel estimation has not been considered in the context of SC-SM systems, we tackle this challenge in this treatise.

To elaborate, substantial research efforts have been invested in conceiving reliable channel estimation algorithms [28]-[30] and pilot transmission strategies [31]-[33] for classic SC transmissions. However, these processes are invarially prone to channel estimation errors. Hence designing robust equalizers by taking into account the channel estimation errors is of salient importance. To expound further, in [34]-[35], robust FDTEs were designed for SC single-input single-output (SISO) systems to cope 
with the channel estimation error by exploiting the characteristics of the specific channel estimator, namely both the channel estimate and the variance of the error, rather than by aiming for achieving a more accurate estimated channel response by conceiving more complicated pilot designs, thus aiming for superiority over their existing nonrobust counterparts. In order to achieve optimal system performance, decision-directed semi-blind channel estimation [36]-[37] was incorporated into our turbo-iterative receivers, in order to yield a robust receiver. Nevertheless, a robust receiver was designed for SM systems relying on imperfect CSI for transmission over Rayleigh flat fading channels [26]. In a nutshell, to retain all the benefits of SC-SM in practical applications relying on realistic imperfect CSI estimation, the design of robust equalization for SC-SM systems becomes an important open issue for future research, which requires further investigations. Against this backdrop, the robust design of FDTEs relying on realistic imperfect channel knowledge and quasi-static Rayleigh fading channel is conceived for SC-SM systems. We design minimum mean-square error (MMSE)-based decision-feedback equalizers (DFEs), since FDTEs impose lower complexities than TDTEs, while attaining considerable multipath diversity gains, where the single-tap based FDTEs can be implemented using the fast Fourier transform (FFT). Additionally, the existing DFE [19] conceived for SC-SM systems was designed based on the assumption of perfect channel knowledge. Therefore, the knowledge of the channel estimator cannot be exploited by the equalizer, not even if the channel estimate and error variance of the estimator are known. In contrast to the original DFE of [19], in this contribution a pair of robust FDTE designs are proposed for SC-SM transmissions, evolved from the classical channel estimation model of [26], [34] as well as from the FDTE structure relying on time-domain (TD) [19], [28], [38] and frequency-domain (FD) [28], [39]-[40] feedback, in order to take into account the specific characteristics of the channel estimator. By considering the practical channel estimation error, the considered system model is different, so the coefficients of the robust TDSDFFDTE and the soft mapper of the equalized signal should be redesigned. Furthermore, decision-directed equalizers are specifically designed for SC-SM systems, which achieve joint robust equalization and data-aided channel estimation (DACE) [36]-[37] to provide a more accurate channel estimate for improving the performance of the proposed robust FDTEs. Compared to the existing robust equalizers and decision-directed equalizers designed for SCSISO systems [34]-[35] by assuming both perfect symbol feedback and unbiased channel estimation, the reliabilities of both the SM feedback symbol and of the biased channel estimation are considered in the proposed robust FDTEs and in the decision-directed SC-SM equalizers advocated, respectively.

Against the above background, the new contributions of this paper are as follows:

1) As a further development of the hybrid DFE structure proposed in [19], [28] and [38], we investigate the joint design of robust time-domain soft-decision feedback (TDSDF) aided FDTE assisted by our channel decoders for practical SC-SM systems relying on realistic imperfect channel knowledge. Furthermore, in this work, the relationship amongst the MSE matrix, the equalized signal and the desired signal is investigated to derive the conditional probability density function (PDF) of the desired SM symbol.

2) To further reduce the complexity imposed, inspired by the receiver structure of FDTE having FD feedback [28], [39]-[40], a robust frequency-domain softdecision feedback (FDSDF) aided FDTE is proposed, where both the feed-forward and feed-back filter are implemented in the FD.

3) In order to further improve the performance of the above pair of robust equalizers by providing a accurate channel estimation, decision-directed equalizers are proposed for reducing the noise-like distortion term embedded in the equalized signal by exploiting the reliable data sequence to refine the channel estimation, where both soft-decision based SM symbols and biased estimation are considered.

4) Based on our extrinsic information transfer (EXIT) chart analysis [41], the convergence behavior of the proposed robust FDTEs is studied. Both the EXIT chart analysis and simulation results confirm that our robust FDTEs provide a better bit error rate (BER) performance than its non-robust counterpart [19].

The remainder of the paper is organized as follows. Section II introduces the system model of SC-SM and our channel estimation model relying on imperfect channel knowledge. In Section III, we propose several robust FDTEs and theoretically derive the coefficients in the presence of channel estimation errors. A joint robust equalization, channel estimation and channel decoding scheme is also investigated in Section III. Section IV characterizes the convergence behavior and the maximum achievable performance of the proposed robust FDTEs based on EXIT chart analysis. While our simulation results and computational complexity comparisons are presented in Section V. Finally, our conclusions are summarized in Section VI.

Notation: $(\cdot)^{T},(\cdot)^{*},(\cdot)^{H}$ and $(\cdot)^{-1}$ refer to the transpose, conjugate, conjugate transpose, and pseudo inverse of a vector/matrix, respectively. $\mathbf{I}_{m}$ represents the $m \times$ $m$-dimensional identity matrix, while $\mathbf{0}_{m \times n}$ denotes the $(m \times n)$-dimensional null matrix having all zero elements. The function $a b s(\cdot)$ denotes the absolute value of all the elements of a matrix, while trace $(\cdot)$ represents the trace of a matrix. Furthermore, diag $(\cdot)$ stands for the operation of reshaping a vector into a diagonal form with all the off-diagonal elements being zeros. The operator $(\cdot) \bmod K$ suggests the modulo- $K$ operation and the operator $\|\cdot\|^{2}$ refers to the Frobenious norm of a vector/matrix. Finally, $E\{\cdot\}$ and $D\{\cdot\}$ represent the mean and the variance of a vector/matrix, respectively.

\section{System Model With Imperfect Channel KNOWLEDGE}

\section{A. System Model}

We consider a broadband SC-SM based MIMO system having $N_{t}$ TAs and $N_{r}$ receive antennas (RAs), communicating over a frequency-selective channel. At the transmit- 
ter, $B=\log _{2} N_{t}+\log _{2} \mathcal{L}$ information bits are transmitted in each time slot, where $\log _{2} N_{t}$ bits are employed to select one out of $N_{t}$ TAs, while $\log _{2} \mathcal{L}$ bits are used for modulating a $\mathcal{L}$-PSK/QAM symbol transmitted by the activated TA. We employ a vectorial notation for representing the $m$ th interval of the transmitted SM symbol, as follows

$$
\mathbf{s}_{m}=[\underbrace{0, \ldots, 0}_{l_{m}-1}, s_{m}, \underbrace{0, \ldots, 0}_{N_{t}-l_{m}}]^{T} \in \mathbb{C}^{N_{t} \times 1},
$$

where $m=0, \ldots, K-1$ represents the index of time interval, while $k=0, \ldots, K-1$ denotes the index of the corresponding frequency subcarrier after the FFT in this paper. Furthermore, $l_{m} \in\{i\}_{i=1}^{N_{t}}$ refers to the index of the activated TA during the $m$ th interval, and $s_{m}$ stands for the $\mathcal{L}$-PSK/QAM symbol transmitted from the classic signal set.

In broadband SC-SM systems, $K$ modulated SM symbols, $\mathbf{s}_{m}$ for $m=0, \ldots, K-1$, are grouped into a block. Then a suitable cyclic prefix (CP) is appended for preventing inter-frame interference. The resultant block is then transmitted through the frequency-selective fading channel having $P$ resolvable multipath links between each TA and RA pair, where the channel model applied here is a quasi-static Rayleigh fading channel. At the receiver, after removing the $\mathrm{CP}$ from each block, the signal received in the $m$ th interval can be expressed by

$$
\mathbf{y}_{m}=\sum_{p=0}^{P-1} \mathbf{H}_{p} \mathbf{s}_{(m-p) \bmod K}+\mathbf{n}_{m}, \text { for } m=0, \ldots, K-1,
$$

where $\mathbf{H}_{p} \in \mathbb{C}^{N_{r} \times N_{t}}$ stands for the channel gain matrix on the $p$ th channel tap, whose elements can be assumed to follow the complex Gaussian distribution of $\mathcal{C N}\left(0, \sigma_{h_{p}}^{2}\right)$, and are independent of each other for different $p$. Furthermore, $\mathbf{n}_{m} \in \mathbb{C}^{N_{r} \times 1}$ represents the additive white Gaussian noise (AWGN), which is composed of i.i.d. $\mathcal{C N}\left(0, \sigma^{2}\right)$ elements. By applying the FFT to both sides of (2), we arrive at

$$
\mathbf{z}_{k}=\mathbf{D}_{k} \mathbf{x}_{k}+\mathbf{w}_{k}, \text { for } k=0, \ldots, K-1,
$$

where $\quad \mathbf{z}_{k}=\frac{1}{\sqrt{K}} \sum_{m=0}^{K-1} \mathbf{y}_{m} e^{-\frac{j 2 \pi m k}{K}}, \quad \mathbf{x}_{k}=$ $\frac{1}{\sqrt{K}} \sum_{m=0}^{K-1} \mathbf{s}_{m} e^{-\frac{j 2 \pi m k}{K}}, \mathbf{D}_{k}=\sum_{p=0}^{P-1} \mathbf{H}_{p} e^{-\frac{j 2 \pi p k}{K}}$, and $\mathbf{w}_{k}=\frac{1}{\sqrt{K}} \sum_{m=0}^{K-1} \mathbf{n}_{m} e^{-\frac{j 2 \pi m k}{K}}$.

Assuming that the TDSDF-FDTE scheme is applied at the receiver, which consists of a feed-forward linear equalizer processed in the FD to combat the influence of the channels, and a feed-back filter implemented in TD to cancel the inter-symbol interference (ISI). As shown in [19], the final equalized signal in the $m$ th interval is given by

$$
\hat{\mathbf{s}}_{m}=\frac{1}{\sqrt{K}} \sum_{k=0}^{K-1} \mathbf{C}_{k} \mathbf{z}_{k} e^{\frac{j 2 \pi m k}{K}}+\mathbf{B} \overline{\mathbf{s}}(m),
$$
where $\overline{\mathbf{s}}(m)=\left[\overline{\mathbf{s}}_{(m-1) \bmod K}^{T}, \overline{\mathbf{s}}_{(m-2) \bmod K}^{T}, \cdots, \overline{\mathbf{s}}_{(m-P+1) \bmod K}^{T}\right.$
and $\overline{\mathbf{s}}_{m}$ represents the soft-feedback SM symbol calculated by the channel decoder, given as

$$
\overline{\mathbf{s}}_{m} \triangleq E\left\{\mathbf{s}_{m}\right\}=\sum_{\boldsymbol{\alpha}_{i} \in \mathbb{S}} \boldsymbol{\alpha}_{i} P\left(\mathbf{s}_{m}=\boldsymbol{\alpha}_{i}\right),
$$

where $\boldsymbol{\alpha}_{i} \in \mathbb{S}$ stands for the transmitted SM vector, and $\mathbb{S}$ represents the SM symbol set. Furthermore, $P\left(\mathbf{s}_{m}=\boldsymbol{\alpha}_{i}\right)$ refers to the probability of the event of $\mathbf{s}_{m}=\boldsymbol{\alpha}_{i}$, which can be expressed by

$$
P\left(\mathbf{s}_{m}=\boldsymbol{\alpha}_{i}\right)=\prod_{n=1}^{B} p\left(c_{n}=b_{n}^{m}\right)=\prod_{n=1}^{B} \frac{e^{\left(b_{n}^{m}\right) L_{a}\left(b_{n}^{m}\right)}}{1+e^{L_{a}\left(b_{n}^{m}\right)}},
$$

where $L_{a}\left(b_{n}^{m}\right)$ represents the a priori log-likelihood ratio (LLR), and $b_{n}^{m} \in\{0,1\}$ stands for the corresponding transmit information bit. According to [19], $\mathbf{C}_{k} \in \mathbb{C}^{N_{t} \times N_{r}}$ and $\mathbf{B} \in \mathbb{C}^{N_{t} \times N_{t}(P-1)}$ in (4) are the coefficients of the feed-forward and feed-back filters, which are derived by assuming perfect CSI. However, in practice the CSI has estimation errors, which degrades the performance of the equalization process [10], [24]-[27].

\section{B. Channel Estimation Model}

In this subsection, the statistical channel estimation model of [26], [34] is employed for expressing the relationship between the real channel $\mathbf{H}_{p}$ and its estimate $\hat{\mathbf{H}}_{p}$ as follows

$$
\hat{\mathbf{H}}_{p}=\mathbf{H}_{p}+\mathbf{H}_{\varepsilon_{p}}
$$

where $\mathbf{H}_{\varepsilon_{p}}$ denotes the channel estimation error, whose elements can be modeled as complex Gaussian random variables with the distribution of $\mathcal{C N}\left(0, \sigma_{\varepsilon_{p}}^{2}\right)$ and are independent for different $p$. It is worth noting that the channel estimation model in (7) closely characterizes practical channel estimation. For example, let us assume that least square (LS) based channel estimation is carried out in the TD to estimate the channel $\hat{\mathbf{H}}_{p}$ and its error covariance $\sigma_{\varepsilon_{p}}^{2}$, where the $N$-length TD unit-power training sequence (TS) of $\left[s_{0}, s_{1}, \cdots, s_{N-1}\right]^{T}, N \geq P$ [42] is employed for estimating the channel impulse response between each TA and RA. Furthermore, a $(P-1)$-sample CP is used. Then, after removing the $\mathrm{CP}$ from the receiver, the signal received from $t$ th $t \in\left(1, N_{t}\right)$ TA and $r$ th $r \in\left(1, N_{r}\right)$ RA pair can be expressed as

$$
\mathbf{y}_{r}=\mathbf{S h}_{t, r}+\mathbf{n}_{r}
$$

where $\mathbf{y}_{r}=\left[y_{r, 0}, y_{r, 1}, \cdots, y_{r, N-1}\right]^{T}$ denotes the signal received by the $r$ th $\mathrm{RA}$, while $\mathbf{S}$ represents an $(N \times P)$ elemetnt Toeplitz matrix, given by

$$
\mathbf{S}=\left[\begin{array}{cccc}
s_{0} & s_{N-1} & \cdots & s_{N-P+1} \\
s_{1} & s_{0} & \cdots & s_{N-P+2} \\
\vdots & \vdots & \ddots & \vdots \\
s_{N-1} & s_{N-2} & \cdots & s_{N-P}
\end{array}\right]
$$

Furthermore, $\mathbf{h}_{t, r}=\left[h_{t, r}^{0}, h_{t, r}^{1}, \cdots, h_{t, r}^{P-1}\right]^{T}$, and $h_{t, r}^{p}$, ]$^{T} p \in(0, P-1)$ represents the element in the $r$ th-row and $t$ th-column of $\mathbf{H}_{p}$. Therefore, the LS estimation can be formulated by $\hat{\mathbf{h}}_{t, r}=\left(\mathbf{S}^{H} \mathbf{S}\right)^{-1} \mathbf{S}^{H} \mathbf{y}_{r}$, with the channel estimation error covariance matrix being

$$
\begin{aligned}
\boldsymbol{\Delta} & \triangleq E\left\{\left(\hat{\mathbf{h}}_{t, r}-\mathbf{h}_{t, r}\right)\left(\hat{\mathbf{h}}_{t, r}-\mathbf{h}_{t, r}\right)^{H}\right\} \\
& =\sigma^{2}\left(\mathbf{S}^{H} \mathbf{S}\right)^{-1}=\frac{\sigma^{2}}{N} \mathbf{I}_{P} .
\end{aligned}
$$




\section{Proposed Robust FDTEs}

\section{A. Proposed Robust TDSDF-FDTE}

1) Coefficients Derivation of Robust TDSDF-FDTE: In this subsection, we first derive the coefficient of the robust TDSDF-FDTE scheme based on the channel estimation model of (7). As observed in [19], imperfect SM symbol feedback and imperfect feedback cancellation occur due to the fact that $\overline{\mathbf{s}}_{m} \neq \mathbf{s}_{m}$, which will lead to a different process than those of conventional robust equalizers relying on assuming having perfect feedback symbols and perfect feedback cancellation [34]-[35]. The architecture of the proposed robust TDSDF-FDTE conceived for our recursive systematic convolutional (RSC)-coded SC-SM system is depicted in Fig. 1, where $\Pi$ and $\Pi^{-1}$ denote the interleaver and de-interleaver, respectively. Furthermore, as shown in Fig. 1, the conditional MSE based on the estimated channel $\hat{\mathbf{H}}$ is invoked for deriving the optimal coefficients of the feed-forward and feed-back filters involved in (4), which can be expressed by

$$
\boldsymbol{\Lambda} \triangleq E\left\{\frac{1}{K} \sum_{m=0}^{K-1}\left(\hat{\mathbf{s}}_{m}-\mathbf{s}_{m}\right)\left(\hat{\mathbf{s}}_{m}-\mathbf{s}_{m}\right)^{H} \mid \hat{\mathbf{H}}\right\} .
$$

By substituting (3) and (4) into (11), and employing Parseval's formula to generate its FD version, we have

$$
\begin{aligned}
\boldsymbol{\Lambda}= & E\left\{\frac{1}{K} \sum_{k=0}^{K-1}\left(\mathbf{C}_{k} \mathbf{D}_{k} \mathbf{x}_{k}+\mathbf{C}_{k} \mathbf{w}_{k}+\hat{\mathbf{B}}_{k} \overline{\mathbf{x}}_{k}-\mathbf{x}_{k}\right)\right. \\
& \left.\left(\mathbf{C}_{k} \mathbf{D}_{k} \mathbf{x}_{k}+\mathbf{C}_{k} \mathbf{w}_{k}+\hat{\mathbf{B}}_{k} \overline{\mathbf{x}}_{k}-\mathbf{x}_{k}\right)^{H} \mid \hat{\mathbf{H}}\right\},
\end{aligned}
$$

where $\hat{\mathbf{B}}_{k}$ and $\overline{\mathbf{x}}_{k}$ correspond to the FFT-based version of $\mathbf{B}$ and $\overline{\mathbf{s}}_{m}$, given by

$$
\hat{\mathbf{B}}_{k}=\mathbf{B F}_{k}, \mathbf{F}_{k}=\left[e^{-\frac{j 2 \pi k}{N}}, \cdots, e^{-\frac{j 2 \pi k(P-1)}{N}}\right]^{T} \otimes \mathbf{I}_{N_{t}},
$$

and

$$
\overline{\mathbf{x}}_{k}=\frac{1}{\sqrt{K}} \sum_{m=0}^{K-1} \overline{\mathbf{s}}_{m} e^{-\frac{j 2 \pi m k}{K}},
$$

respectively.

As observed in (12), the key step to derive the MSE matrix $\boldsymbol{\Lambda}$ is to find $E\left\{\mathbf{D}_{k} \mid \hat{\mathbf{H}}\right\}$ and $E\left\{\mathbf{D}_{k} \mathbf{D}_{k}^{H} \mid \hat{\mathbf{H}}\right\}$. Since $\mathbf{D}_{k}$ is the FFT-based version of $\hat{\mathbf{H}}_{p}$, we have

$$
\begin{aligned}
\hat{\mathbf{D}}_{k} & \triangleq E\left\{\mathbf{D}_{k} \mid \hat{\mathbf{H}}\right\}=\sum_{p=0}^{P-1} E\left\{\mathbf{H}_{p} \mid \hat{\mathbf{H}}\right\} e^{-\frac{j 2 \pi p k}{K}} \\
& =\sum_{p=0}^{P-1} \hat{\mathbf{H}}_{p} e^{-\frac{j 2 \pi p k}{K}},
\end{aligned}
$$

and

$$
\begin{aligned}
& E\left\{\mathbf{D}_{k} \mathbf{D}_{k}^{H} \mid \hat{\mathbf{H}}\right\} \\
& =E\left\{\sum_{p=0}^{P-1} \mathbf{H}_{p} e^{-\frac{j 2 \pi p k}{K}} \sum_{l=0}^{P-1} \mathbf{H}_{l}^{H} e^{\frac{j 2 \pi l k}{K}} \mid \hat{\mathbf{H}}\right\} \\
& =E\left\{\sum_{p=0}^{P-1} \sum_{l=0}^{P-1}\left(\hat{\mathbf{H}}_{p}-\mathbf{H}_{\varepsilon_{p}}\right)\left(\hat{\mathbf{H}}_{l}^{H}-\mathbf{H}_{\varepsilon_{l}}^{H}\right) e^{\frac{j 2 \pi(l-p) k}{K}} \mid \hat{\mathbf{H}}\right\} \\
& =\sum_{p=0}^{P-1} \sum_{l=0}^{P-1} \hat{\mathbf{H}}_{p} \hat{\mathbf{H}}_{l}^{H} e^{\frac{j 2 \pi(l-p) k}{K}}+\sum_{p=0}^{P-1} E\left\{\mathbf{H}_{\varepsilon_{p}} \mathbf{H}_{\varepsilon_{p}}^{H} \mid \hat{\mathbf{H}}\right\} \\
& =\hat{\mathbf{D}}_{k} \hat{\mathbf{D}}_{k}^{H}+N_{t} \sum_{p=0}^{P-1} \sigma_{\varepsilon_{p}}^{2} \mathbf{I}_{N_{r}} .
\end{aligned}
$$

Substituting $E\left\{\mathbf{D}_{k} \mid \hat{\mathbf{H}}\right\}$ and $E\left\{\mathbf{D}_{k} \mathbf{D}_{k}^{H} \mid \hat{\mathbf{H}}\right\}$ into the MSE matrix of (12), we arrive at

$$
\begin{aligned}
\boldsymbol{\Lambda} & =\frac{1}{K} \sum_{k=0}^{K-1}\left(\mathbf{C}_{k} \hat{\mathbf{D}}_{k}-\mathbf{I}_{N_{t}}\right) E\left\{\mathbf{x}_{k} \mathbf{x}_{k}^{H}\right\}\left(\hat{\mathbf{D}}_{k}^{H} \mathbf{C}_{k}^{H}-\mathbf{I}_{N_{t}}\right) \\
& +\left(\mathbf{C}_{k} \hat{\mathbf{D}}_{k}-\mathbf{I}_{N_{t}}\right) E\left\{\mathbf{x}_{k} \overline{\mathbf{x}}_{k}^{H}\right\} \hat{\mathbf{B}}_{k}^{H}+\hat{\mathbf{B}}_{k} E\left\{\overline{\mathbf{x}}_{k} \overline{\mathbf{x}}_{k}^{H}\right\} \hat{\mathbf{B}}_{k}^{H} \\
& +\hat{\mathbf{B}}_{k} E\left\{\overline{\mathbf{x}}_{k} \mathbf{x}_{k}^{H}\right\}\left(\hat{\mathbf{D}}_{k}^{H} \mathbf{C}_{k}^{H}-\mathbf{I}_{N_{t}}\right)+\hat{\sigma}^{2} \mathbf{C}_{k} \mathbf{C}_{k}^{H},
\end{aligned}
$$

where

$$
\hat{\sigma}^{2}=\operatorname{trace}\left(E\left\{\mathbf{x}_{k} \mathbf{x}_{k}^{H}\right\}\right) \sum_{p=0}^{P-1} \sigma_{\varepsilon_{p}}^{2}+\sigma^{2} .
$$

Furthermore, $E\left\{\mathbf{x}_{k} \mathbf{x}_{k}^{H}\right\}$ is the diagonal second-order moment matrix of the FD symbol $\mathbf{x}_{k}$, given by

$$
\begin{aligned}
& E\left\{\mathbf{x}_{k} \mathbf{x}_{k}^{H}\right\}=\frac{1}{K} \sum_{m=0}^{K-1} E\left\{\mathbf{s}_{m} \mathbf{s}_{m}^{H}\right\}, \\
& E\left\{\mathbf{s}_{m} \mathbf{s}_{m}^{H}\right\}=\sum_{\boldsymbol{\alpha}_{i} \in \mathbb{S}} P\left(\mathbf{s}_{k}=\boldsymbol{\alpha}_{i}\right) \boldsymbol{\alpha}_{i} \boldsymbol{\alpha}_{i}{ }^{H} .
\end{aligned}
$$

Next, the approximation of the reliability of feedback decision $E\left\{\mathbf{s}_{m} \overline{\mathbf{s}}_{m}^{H}\right\} \approx E\left\{\mathbf{s}_{m} E\left\{\mathbf{s}_{m}\right\}^{H}\right\}=E\left\{\mathbf{s}_{m}\right\} E\left\{\mathbf{s}_{m}\right\}^{H}=$ $\overline{\mathbf{s}}_{m} \overline{\mathbf{s}}_{m}^{H}$ defined in [19] is employed, yielding

$E\left\{\overline{\mathbf{x}}_{k} \mathbf{x}_{k}^{H}\right\}=E\left\{\mathbf{x}_{k} \overline{\mathbf{x}}_{k}^{H}\right\}=E\left\{\overline{\mathbf{x}}_{k} \overline{\mathbf{x}}_{k}^{H}\right\}=\frac{1}{K} \sum_{m=0}^{K-1} \overline{\mathbf{s}}_{m} \overline{\mathbf{s}}_{m}^{H}$

Setting the derivative of (17) with respect to $\mathbf{C}_{k}$ to zero, we have the optimal feed-forward filter as follows

$$
\begin{aligned}
\mathbf{C}_{k}= & \left(E\left\{\mathbf{x}_{k} \mathbf{x}_{k}^{H}\right\}-\hat{\mathbf{B}}_{k} E\left\{\overline{\mathbf{x}}_{k} \mathbf{x}_{k}^{H}\right\}\right) \\
& \hat{\mathbf{D}}_{k}^{H}\left(\hat{\mathbf{D}}_{k} E\left\{\mathbf{x}_{k} \mathbf{x}_{k}^{H}\right\} \hat{\mathbf{D}}_{k}^{H}+\hat{\sigma}^{2} \mathbf{I}_{N_{r}}\right)^{-1} \\
= & \left(E\left\{\mathbf{x}_{k} \mathbf{x}_{k}^{H}\right\}-\hat{\mathbf{B}}_{k} E\left\{\overline{\mathbf{x}}_{k} \mathbf{x}_{k}^{H}\right\}\right) \\
& \left(\hat{\mathbf{D}}_{k}^{H} \hat{\mathbf{D}}_{k} E\left\{\mathbf{x}_{k} \mathbf{x}_{k}^{H}\right\}+\hat{\sigma}^{2} \mathbf{I}_{N_{t}}\right)^{-1} \hat{\mathbf{D}}_{k}^{H} .
\end{aligned}
$$

Similarly, by setting the derivative of (17) with respect to B to zero after substituting (21) back into (17), we have the optimal feed-back filter as follows

$$
\begin{aligned}
\mathbf{B}= & {\left[\sum_{k=0}^{K-1} \hat{\sigma}^{2}\left(\hat{\mathbf{D}}_{k}^{H} \hat{\mathbf{D}}_{k} E\left\{\mathbf{x}_{k} \mathbf{x}_{k}^{H}\right\}+\hat{\sigma}^{2} \mathbf{I}_{N_{t}}\right)^{-1} \mathbf{F}_{k}^{H}\right] } \\
& \left\{\sum _ { k = 0 } ^ { K - 1 } \mathbf { F } _ { k } \left[\hat { \sigma } ^ { 2 } E \{ \overline { \mathbf { x } } _ { k } \mathbf { x } _ { k } ^ { H } \} E \{ \mathbf { x } _ { k } \mathbf { x } _ { k } ^ { H } \} ^ { - 1 } \left(\hat{\mathbf{D}}_{k}^{H} \hat{\mathbf{D}}_{k} E\left\{\mathbf{x}_{k} \mathbf{x}_{k}^{H}\right\}\right.\right.\right. \\
+ & \left.\left.\left.\hat{\sigma}^{2} \mathbf{I}_{N_{t}}\right)^{-1}+\mathbf{I}_{N_{t}}-E\left\{\overline{\mathbf{x}}_{k} \mathbf{x}_{k}^{H}\right\} E\left\{\mathbf{x}_{k} \mathbf{x}_{k}^{H}\right\}^{-1}\right] \mathbf{F}_{k}^{H}\right\}^{-1} .
\end{aligned}
$$

It is worth noting that the above derivation in the robust TDSDF-FDTE design is quite different from that of the conventional TDSDF-FDTE of [19], since the conventional equalizer only directly replaces the real channel $\mathbf{D}_{k}$ by the estimated one $\hat{\mathbf{D}}_{k}$, where both the estimated channel $\hat{\mathbf{D}}_{k}$ and the second-order moment matrix $E\left\{\mathbf{D}_{k} \mathbf{D}_{k}^{H} \mid \hat{\mathbf{H}}\right\}$ are utilized in the design of the robust TDSDF-FDTE. Thus, a more robust system performance can be achieved by the proposed equalizer.

2) Output Extrinsic LLR of Equalized Signal: Given the optimal filter coefficients derived in (21) and (22), the equalized signal $\hat{\mathbf{s}}_{m}$ in (4), which is an estimate of the corresponding desired SM symbol $\mathbf{s}_{m}$, has to be converted to the corresponding extrinsic LLRs (defined by the posteriori LLR minus the priori LLR [43]) of the transmitted information bits $b_{n}^{m}, n \in\{1, B\}$ for the channel decoder, where the calculation of the conditional PDF of the equalized signal $\hat{\mathbf{s}}_{m}$ is needed. In contrast to the LLRs computed in [19] under the perfect CSI assumption, in the case of imperfect channel estimation, the inputs of the channel decoder in terms of the LLRs can be calculated as follows. Firstly, we rewrite the equalized signal of (4) in the TD circular convolution form, as follows

$$
\begin{aligned}
\hat{\mathbf{s}}_{m} & =\sum_{p=0}^{K-1} \mathbf{G}_{p} \mathbf{s}_{(m-p) \bmod K}+\sum_{p=1}^{P-1} \mathbf{B}_{p} \overline{\mathbf{s}}_{(m-p) \bmod K} \\
& +\frac{1}{\sqrt{K}} \sum_{k=0}^{K-1} \mathbf{C}_{k} \mathbf{w}_{k} e^{\frac{j 2 \pi m k}{K}},
\end{aligned}
$$




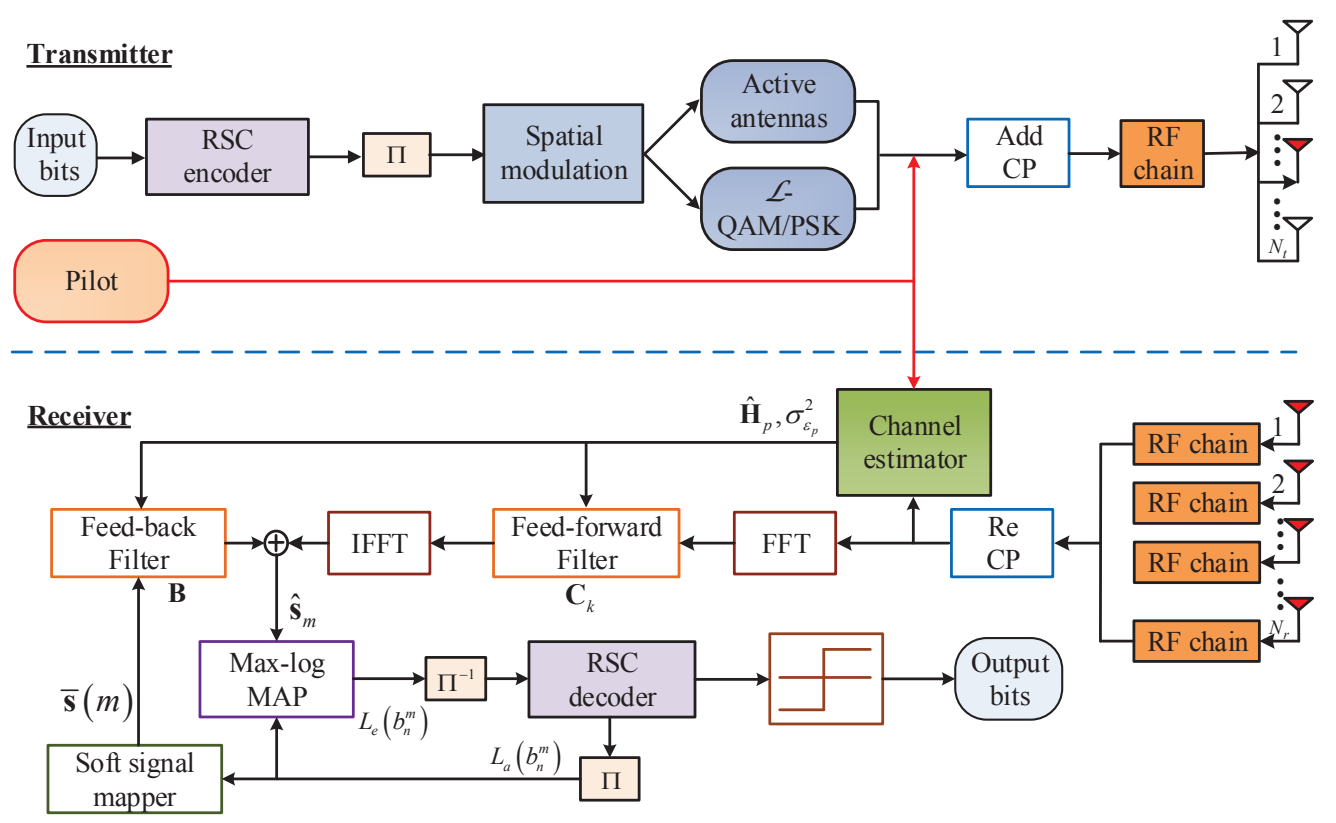

Fig. 1. Transceiver architecture of the proposed robust TDSDF-FDTE aided SC-SM scheme with imperfect channel knowledge.

where $\mathbf{B} \triangleq\left[\mathbf{B}_{1}, \cdots, \mathbf{B}_{P-1}\right]$ and $\mathbf{G}_{p} \triangleq$ $\frac{1}{K} \sum_{k=0}^{K-1} \mathbf{C}_{k} \mathbf{D}_{k} e^{\frac{j 2 \pi p k}{K}}$. In the case of perfect channel knowledge, by assuming that the distortion term containing both the remaining ISI and noise is Gaussian distributed [19], the equalized signal can be directly represented by a Gaussian distribution of $\mathcal{C N}\left(E\left\{\hat{\mathbf{s}}_{m} \mid \mathbf{s}_{m}, \mathbf{H}\right\}, D\left\{\hat{\mathbf{s}}_{m} \mid \mathbf{s}_{m}, \mathbf{H}\right\}\right)$. However, this approach is unsuitable for the case of imperfect channel knowledge, because the coefficient matrix $\mathbf{G}_{0}$ associated with the desired SM symbol $\mathbf{s}_{m}$ in (23) is related to the real channel $\mathbf{H}$, which is not perfectly known at the receiver. To solve this problem, the relationship amongst the MSE matrix, the equalized signal and the desired signal in (11) is investigated in order to calculate the conditional PDF of the equalized signal $\hat{\mathbf{s}}_{m}$ in (4). According to (23), we can rewrite the equalized signal as follows

$$
\hat{\mathbf{s}}_{m}=E\left\{\mathbf{G}_{0} \mid \hat{\mathbf{H}}\right\} \mathbf{s}_{m}+\mathbf{U}_{m}=\hat{\mathbf{G}}_{0} \mathbf{s}_{m}+\mathbf{U}_{m},
$$

where $\hat{\mathbf{G}}_{0}$ constitutes a multiplier of the desired signal, given by

$$
\hat{\mathbf{G}}_{0} \triangleq E\left\{\mathbf{G}_{0} \mid \hat{\mathbf{H}}\right\}=\frac{1}{K} \sum_{k=0}^{K-1} \mathbf{C}_{k} \hat{\mathbf{D}}_{k} .
$$

Furthermore, $\mathbf{U}_{m}$ represents the distortion term, which can be expressed by

$$
\begin{aligned}
\mathbf{U}_{m} & =\sum_{p=1}^{K-1} \mathbf{G}_{p} \mathbf{s}_{(m-p) \bmod K}+\sum_{p=1}^{P-1} \mathbf{B}_{p} \overline{\mathbf{s}}_{(m-p) \bmod K} \\
& +\frac{1}{\sqrt{K}} \sum_{k=0}^{K-1} \mathbf{C}_{k} \mathbf{w}_{k} e^{\frac{j 2 \pi m k}{K}}+\left(\mathbf{G}_{0}-\hat{\mathbf{G}}_{0}\right) \mathbf{s}_{m} .
\end{aligned}
$$

It can be observed in (26) that the distortion term $\mathbf{U}_{m}$ contains not only the residual ISI and filtered noise, but also a noise-like distortion component due to the channel estimation error. Again, let us assume that $\mathbf{U}_{m}$ is Gaussian distributed with zero mean $\mathbf{0}_{N_{t} \times 1}$, which is independent of the desired signal. By substituting the equalized signal $\hat{\mathbf{s}}_{m}$ of (24) into the MSE matrix $\boldsymbol{\Lambda}$ of (11), we can refine
$\Lambda$ as follows

$$
\begin{aligned}
\boldsymbol{\Lambda} & =E\left\{\frac{1}{K} \sum_{m=0}^{K-1}\left(\hat{\mathbf{G}}_{0} \mathbf{s}_{m}+\mathbf{U}_{m}-\mathbf{s}_{m}\right)\left(\hat{\mathbf{G}}_{0} \mathbf{s}_{m}+\mathbf{U}_{m}-\mathbf{s}_{m}\right)^{H} \mid \hat{\mathbf{H}}\right\} \\
& =\left(\hat{\mathbf{G}}_{0}-\mathbf{I}_{N_{t}}\right) \frac{1}{K} \sum_{m=0}^{K-1} E\left\{\mathbf{s}_{m} \mathbf{s}_{m}^{H}\right\}\left(\hat{\mathbf{G}}_{0}-\mathbf{I}_{N_{t}}\right)^{H} \\
& +\frac{1}{K} \sum_{m=0}^{K-1} E\left\{\mathbf{U}_{m} \mathbf{U}_{m}^{H} \mid \hat{\mathbf{H}}\right\} \\
& =\left(\hat{\mathbf{G}}_{0}-\mathbf{I}_{N_{t}}\right) E\left\{\mathbf{x}_{k} \mathbf{x}_{k}^{H}\right\}\left(\hat{\mathbf{G}}_{0}-\mathbf{I}_{N_{t}}\right)^{H}+E\left\{\mathbf{U}_{m} \mathbf{U}_{m}^{H} \mid \hat{\mathbf{H}}\right\},
\end{aligned}
$$

where

$$
\begin{aligned}
E\left\{\mathbf{U}_{0} \mathbf{U}_{0}^{H} \mid \hat{\mathbf{H}}\right\} & =\cdots=E\left\{\mathbf{U}_{K-1} \mathbf{U}_{K-1}^{H} \mid \hat{\mathbf{H}}\right\} \\
& =\frac{1}{K} \sum_{m=0}^{K-1} E\left\{\mathbf{U}_{m} \mathbf{U}_{m}^{H} \mid \hat{\mathbf{H}}\right\} .
\end{aligned}
$$

Then based on (27), the second-order moment matrix of $\mathbf{U}_{m}$ can be derived as

$$
E\left\{\mathbf{U}_{m} \mathbf{U}_{m}^{H} \mid \hat{\mathbf{H}}\right\}=\boldsymbol{\Lambda}-\left(\hat{\mathbf{G}}_{0}-\mathbf{I}_{N_{t}}\right) E\left\{\mathbf{x}_{k} \mathbf{x}_{k}^{H}\right\}\left(\hat{\mathbf{G}}_{0}-\mathbf{I}_{N_{t}}\right)^{H}
$$

where the MSE matrix $\boldsymbol{\Lambda}$ can be expressed with the aid of its FD version in (12), yielding

$$
\begin{aligned}
\boldsymbol{\Lambda} & =E\left\{\mathbf{x}_{k} \mathbf{x}_{k}^{H}\right\}-E\left\{\mathbf{x}_{k} \mathbf{x}_{k}^{H}\right\} \hat{\mathbf{G}}_{0}+\frac{1}{K} \sum_{k=0}^{K-1} \mathbf{C}_{k} \hat{\mathbf{D}}_{k} E\left\{\mathbf{x}_{k} \overline{\mathbf{x}}_{k}^{H}\right\} \hat{\mathbf{B}}_{k}^{H} \\
& +\hat{\mathbf{B}}_{k} E\left\{\mathbf{x}_{k} \overline{\mathbf{x}}_{k}^{H}\right\} \hat{\mathbf{B}}_{k}^{H}-\hat{\mathbf{B}}_{k} E\left\{\mathbf{x}_{k} \overline{\mathbf{x}}_{k}^{H}\right\}-E\left\{\mathbf{x}_{k} \overline{\mathbf{x}}_{k}^{H}\right\} \hat{\mathbf{B}}_{k}^{H} .
\end{aligned}
$$

Finally, according to (24), (25) and (29), the extrinsic LLR at the output of the proposed robust equalizer and entered into the channel decoder can be calculated by the wellknown Max-Log maximum a posterior (MAP) algorithm [19], [43], as follows

$$
\begin{aligned}
& L_{e}\left(b_{n}^{m}\right)=\max _{\mathbf{s}_{m} \in \mathbb{S}_{1}^{n}}\left[\frac{-\left\|\hat{\mathbf{s}}_{m}-\hat{\mathbf{G}}_{0} \mathbf{s}_{m}\right\|^{2}}{\sigma_{m}^{2}}+\sum_{j \neq n}^{B} b_{j}^{m} L_{a}\left(b_{j}^{m}\right)\right] \\
& -\max _{\mathbf{s}_{m} \in \mathbb{S}_{0}^{n}}\left[\frac{-\left\|\hat{\mathbf{s}}_{m}-\hat{\mathbf{G}}_{0} \mathbf{s}_{m}\right\|^{2}}{\sigma_{m}^{2}}+\sum_{j \neq n}^{B} b_{j}^{m} L_{a}\left(b_{j}^{m}\right)\right],
\end{aligned}
$$

where $\mathbb{S}_{0}^{n}$ and $\mathbb{S}_{1}^{n}$ refer to the legitimate subspaces of $\mathrm{SM}$ symbols, satisfying $\mathbb{S}_{0}^{n}=\left\{\mathbf{x}_{k} \in \mathbb{S}^{n}: b_{n}=0\right\}$ and $\mathbb{S}_{1}^{n}=\left\{\mathbf{x}_{k} \in \mathbb{S}^{n}: b_{n}=1\right\}$, respectively. Here, $\sigma_{m}^{2}$ stands for 
the covariance value of $E\left\{\mathbf{U}_{m} \mathbf{U}_{m}^{H} \mid \hat{\mathbf{H}}\right\}$ in (29), which is expressed as:

$$
\sigma_{m}^{2}=\frac{\operatorname{trace}\left[a b s\left(E\left\{\mathbf{U}_{m} \mathbf{U}_{m}^{H} \mid \hat{\mathbf{H}}\right\}\right)\right]}{N_{t}} .
$$

\section{B. Proposed Robust FDSDF-FDTE}

1) Proposed Robust FDSDF-FDTE design: In the proposed robust TDSDF-FDTE design, the operation of the $N_{t}(P-1)$-dimensional matrix inversion involved in the calculation of feed-back filter imposes a high computational complexity. To tackle this issue, a robust FDSDFFDTE designed for SC-SM is proposed in Fig. 2, where both the feed-forward and feed-back filter are implemented in the FD. In the proposed robust FDSDF-FDTE, the FD single-tap based feed-back filter is conceived rather than the TD multiple-taps based feed-back filter, leading to a lower computational complexity than the robust TDSDF-FDTE. Moveover, imperfect SM symbol feedback and imperfect feedback cancellation are assumed in the proposed robust FDSDF-FDTE. As shown in Fig. 2, the final equalized signal $\hat{\mathbf{s}}_{m}$ in the $m$ th symbol interval is given by

$$
\hat{\mathbf{s}}_{m}=\frac{1}{\sqrt{K}} \sum_{k=0}^{K-1}\left(\mathbf{C}_{k} \mathbf{z}_{k}+\hat{\mathbf{B}}_{k} \overline{\mathbf{x}}_{k}\right) e^{\frac{j 2 \pi m k}{K}},
$$

where $\overline{\mathbf{x}}_{k}$ corresponds to the FFT-based version of $\overline{\mathbf{s}}_{m}$ defined in (14).

Here, $\mathbf{C}_{k} \in \mathbb{C}^{N_{t} \times N_{r}}$ and $\hat{\mathbf{B}}_{k} \in \mathbb{C}^{N_{t} \times N_{t}}$ are the coefficients of the feed-forward and feed-back filters, designed by solving the following optimization

$$
\begin{array}{ll}
\underset{\mathbf{C}_{k} \in \mathbb{C}^{N_{t} \times N_{r}}}{\arg \min }, & \boldsymbol{\Lambda} \\
\hat{\mathbf{B}}_{k} \in \mathbb{C}^{N_{t} \times N_{t}} & \\
\text { subject to } & \sum_{k=0}^{K-1} \hat{\mathbf{B}}_{k}=\mathbf{0}_{N_{t} \times N_{t}},
\end{array}
$$

where $\boldsymbol{\Lambda}$ is the conditional MSE based on the estimated channel $\hat{\mathbf{H}}$, defined in (11). Here, the constraint $\sum_{k=0}^{K-1} \hat{\mathbf{B}}_{k}=\mathbf{0}_{N_{t} \times N_{t}}$ ensures that the feed-back filter does not remove the desired component [44]-[45]. According to the classic Lagrangian method, $\mathbf{C}_{k}$ and $\hat{\mathbf{B}}_{k}$ are the solutions of the following equations

$$
\left\{\begin{array}{l}
\frac{\partial\left(\boldsymbol{\Lambda}+\mathbf{A} \sum_{k=0}^{K-1} \hat{\mathbf{B}}_{k}\right)}{\partial \mathbf{C}_{k-1}}=\mathbf{0}_{N_{r} \times N_{t}} \\
\frac{\partial\left(\boldsymbol{\Lambda}+\mathbf{A} \sum_{k=0}^{K-1} \hat{\mathbf{B}}_{k}\right)}{\partial \hat{\mathbf{B}}_{k}}=\mathbf{0}_{N_{t} \times N_{t}} \\
\sum_{k=0}^{K-1} \hat{\mathbf{B}}_{k}=\mathbf{0}_{N_{t} \times N_{t}}
\end{array},\right.
$$

where $\mathbf{A}$ is the Lagrange multiplier. Substituting the FD version of $\boldsymbol{\Lambda}$ of (17) into (35), we have the optimal coefficients of the feed-forward and feed-back filters as follows

$$
\begin{aligned}
\mathbf{C}_{k}= & \left(E\left\{\mathbf{x}_{k} \mathbf{x}_{k}^{H}\right\}-\hat{\mathbf{B}}_{k} E\left\{\overline{\mathbf{x}}_{k} \mathbf{x}_{k}^{H}\right\}\right) \\
& \left(\hat{\mathbf{D}}_{k}^{H} \hat{\mathbf{D}}_{k} E\left\{\mathbf{x}_{k} \mathbf{x}_{k}^{H}\right\}+\hat{\sigma}^{2} \mathbf{I}_{N_{t}}\right)^{-1} \hat{\mathbf{D}}_{k}^{H},
\end{aligned}
$$

and

$$
\begin{aligned}
\hat{\mathbf{B}}_{k}= & {\left[\hat{\sigma}^{2}\left(\hat{\mathbf{D}}_{k}^{H} \hat{\mathbf{D}}_{k} E\left\{\mathbf{x}_{k} \mathbf{x}_{k}^{H}\right\}+\hat{\sigma}^{2} \mathbf{I}_{N_{t}}\right)^{-1}-K \mathbf{A}^{H} E\left\{\overline{\mathbf{x}}_{k} \mathbf{x}_{k}^{H}\right\}^{-1}\right] } \\
& {\left[\hat{\sigma}^{2} E\left\{\overline{\mathbf{x}}_{k} \mathbf{x}_{k}^{H}\right\} E\left\{\mathbf{x}_{k} \mathbf{x}_{k}^{H}\right\}^{-1}\left(\hat{\mathbf{D}}_{k}^{H} \hat{\mathbf{D}}_{k} E\left\{\mathbf{x}_{k} \mathbf{x}_{k}^{H}\right\}+\hat{\sigma}^{2} \mathbf{I}_{N_{t}}\right)\right.} \\
+ & \left.\mathbf{I}_{N_{t}}-E\left\{\overline{\mathbf{x}}_{k} \mathbf{x}_{k}^{H}\right\} E\left\{\mathbf{x}_{k} \mathbf{x}_{k}^{H}\right\}^{-1}\right]^{-1},
\end{aligned}
$$

where $E\left\{\mathbf{x}_{k} \mathbf{x}_{k}^{H}\right\}$ and $E\left\{\overline{\mathbf{x}}_{k} \mathbf{x}_{k}^{H}\right\}$ are defined in (19) and (20), respectively. Furthermore, $\hat{\mathbf{D}}_{k}$ and $\hat{\sigma}^{2}$ are defined in (15) and (18), respectively. Here, $K \mathbf{A}^{H} E\left\{\overline{\mathbf{x}}_{k} \mathbf{x}_{k}^{H}\right\}^{-1}$ is a constant matrix for a given index $k$, which is given in (38). As observed in (37), only the $N_{t}$-dimensional matrix inversion is used, rather than the $N_{t}(P-1)$-dimensional matrix inversion involved in the calculation of the feedback filter in the proposed robust TDSDF-FDTE, hence leading to a reduced computational complexity.

2) Output Extrinsic LLR of Equalized Signal: Similar to the soft output of the robust TDSDF-FDTE presented in Section III-A 2, the soft mapper of the proposed robust FDSDF-FDTE should also exploit the relationship amongst the MSE matrix, the equalized signal and the desired signal in (11) for determining the conditional PDF of the equalized signal $\hat{\mathbf{s}}_{m}$ of (33). Firstly, the equalized signal $\hat{\mathbf{s}}_{m}$ in (33) can be refined by invoking the TD circular convolution form, as follows

$$
\begin{aligned}
\hat{\mathbf{s}}_{m} & =\sum_{p=0}^{K-1} \mathbf{G}_{p} \mathbf{s}_{(m-p) \bmod K}+\sum_{p=0}^{K-1} \mathbf{B}_{p} \overline{\mathbf{s}}_{(m-p) \bmod K} \\
& +\frac{1}{\sqrt{K}} \sum_{k=0}^{K-1} \mathbf{C}_{k} \mathbf{w}_{k} e^{\frac{j 2 \pi m k}{K}},
\end{aligned}
$$

where $\mathbf{G}_{p} \triangleq \frac{1}{K} \sum_{k=0}^{K-1} \mathbf{C}_{k} \mathbf{D}_{k} e^{\frac{j 2 \pi p k}{K}}$ and $\mathbf{B}_{p} \triangleq$ $\frac{1}{K} \sum_{k=0}^{K-1} \hat{\mathbf{B}}_{k} e^{\frac{j 2 \pi p k}{K}}$. Then the equalized signal can be rewritten as the desired signal plus a distortion term as follows

$$
\hat{\mathbf{s}}_{m}=E\left\{\mathbf{G}_{0} \mid \hat{\mathbf{H}}\right\} \mathbf{s}_{m}+\mathbf{U}_{m}=\hat{\mathbf{G}}_{0} \mathbf{s}_{m}+\mathbf{U}_{m},
$$

where the multiplier of the desired signal $\hat{\mathbf{G}}_{0}$ and the distortion term $\mathbf{U}_{m}$ are formulated as

$$
\hat{\mathbf{G}}_{0} \triangleq E\left\{\mathbf{G}_{0} \mid \hat{\mathbf{H}}\right\}=\frac{1}{K} \sum_{k=0}^{K-1} \mathbf{C}_{k} \hat{\mathbf{D}}_{k} .
$$

and

$$
\begin{aligned}
\mathbf{U}_{m} & =\sum_{p=1}^{K-1} \mathbf{G}_{p} \mathbf{s}_{(m-p) \bmod K}+\sum_{p=0}^{K-1} \mathbf{B}_{p} \overline{\mathbf{s}}_{(m-p) \bmod K} \\
& +\frac{1}{\sqrt{K}} \sum_{k=0}^{K-1} \mathbf{C}_{k} \mathbf{w}_{k} e^{\frac{j 2 \pi m k}{K}}+\left(\mathbf{G}_{0}-\hat{\mathbf{G}}_{0}\right) \mathbf{s}_{m},
\end{aligned}
$$

respectively. Therefore, similar to (29), the second-order moment matrix of $\mathbf{U}_{m}$ can still be derived by

$E\left\{\mathbf{U}_{m} \mathbf{U}_{m}^{H} \mid \hat{\mathbf{H}}\right\}=\boldsymbol{\Lambda}-\left(\hat{\mathbf{G}}_{0}-\mathbf{I}_{N_{t}}\right) E\left\{\mathbf{x}_{k} \mathbf{x}_{k}^{H}\right\}\left(\hat{\mathbf{G}}_{0}-\mathbf{I}_{N_{t}}\right)^{H}$,

where the MSE matrix $\boldsymbol{\Lambda}$ can be obtained by substituting (36), (37) into (30). Finally, the extrinsic information based on the equalized signal $\hat{\mathbf{s}}_{m}$ output by the robust equalizer and entered into the channel decoder can be obtained by (31) and (32).

\section{Proposed Decision-Directed Robust Equalizers}

In the proposed pair of robust nonlinear FDTE designs of the previous subsections, a component related to the channel estimation error was identified in the distortion term of (26) and (42). Therefore the system performance is still limited by the quality of the channel estimation. Hence, better system performance can be attained by improving the accuracy of the channel estimation. In this ${ }^{1}$ subsection, decision-directed equalizers are proposed by using joint robust equalization and DACE for reducing the noise-like distortion term embedded in the equalized signal. The architecture of the proposed decision-directed 


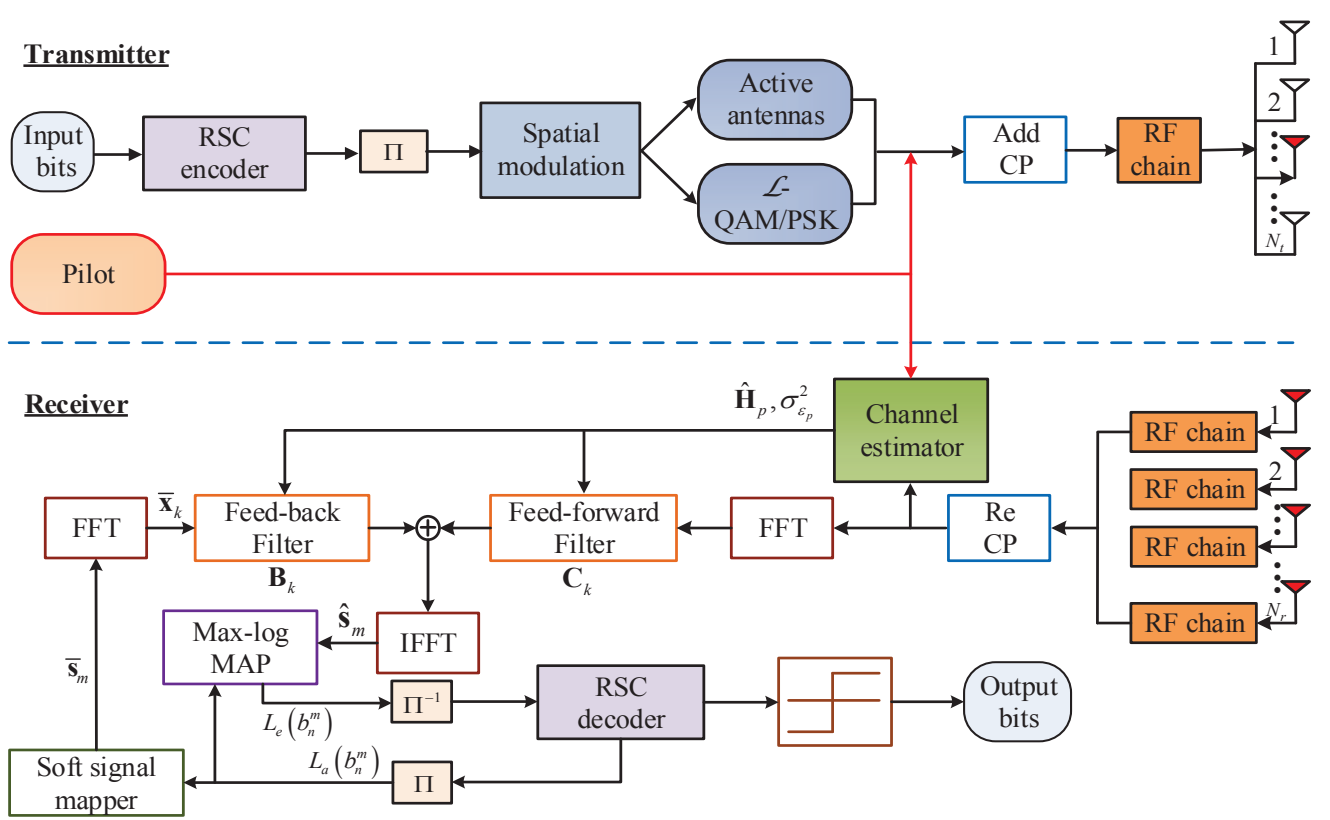

Fig. 2. Transceiver architecture of the proposed robust FDSDF-FDTE aided SC-SM scheme with imperfect channel knowledge.

$$
\begin{aligned}
K \mathbf{A}^{H} E\left\{\overline{\mathbf{x}}_{k} \mathbf{x}_{k}^{H}\right\}^{-1}= & \left\{\sum _ { k = 0 } ^ { K - 1 } \hat { \sigma } ^ { 2 } ( \hat { \mathbf { D } } _ { k } ^ { H } \hat { \mathbf { D } } _ { k } E \{ \mathbf { x } _ { k } \mathbf { x } _ { k } ^ { H } \} + \hat { \sigma } ^ { 2 } \mathbf { I } _ { N _ { t } } ) ^ { - 1 } \left[\hat{\sigma}^{2} E\left\{\overline{\mathbf{x}}_{k} \mathbf{x}_{k}^{H}\right\} E\left\{\mathbf{x}_{k} \mathbf{x}_{k}^{H}\right\}^{-1}\right.\right. \\
& \left.\left.\left(\hat{\mathbf{D}}_{k}^{H} \hat{\mathbf{D}}_{k} E\left\{\mathbf{x}_{k} \mathbf{x}_{k}^{H}\right\}+\hat{\sigma}^{2} \mathbf{I}_{N_{t}}\right)^{-1}+\mathbf{I}_{N_{t}}-E\left\{\overline{\mathbf{x}}_{k} \mathbf{x}_{k}^{H}\right\} E\left\{\mathbf{x}_{k} \mathbf{x}_{k}^{H}\right\}^{-1}\right]^{-1}\right\} \\
& \left\{\sum _ { k = 0 } ^ { K - 1 } \left[\hat{\sigma}^{2} E\left\{\overline{\mathbf{x}}_{k} \mathbf{x}_{k}^{H}\right\} E\left\{\mathbf{x}_{k} \mathbf{x}_{k}^{H}\right\}^{-1}\left(\hat{\mathbf{D}}_{k}^{H} \hat{\mathbf{D}}_{k} E\left\{\mathbf{x}_{k} \mathbf{x}_{k}^{H}\right\}+\hat{\sigma}^{2} \mathbf{I}_{N_{t}}\right)^{-1}\right.\right. \\
& \left.\left.+\mathbf{I}_{N_{t}}-E\left\{\overline{\mathbf{x}}_{k} \mathbf{x}_{k}^{H}\right\} E\left\{\mathbf{x}_{k} \mathbf{x}_{k}^{H}\right\}^{-1}\right]^{-1}\right\}^{-1} .
\end{aligned}
$$

robust equalizers is depicted in Fig. 3. As observed in Fig. 3 , two main steps are invoked for improving the accuracy of the channel estimation: 1) the conditional first- and second-order moment matrices based on the a priori MSE channel estimation results are invoked for alleviating the influence of the channel estimation error before the process of robust equalization, and 2) the output soft-decision SM symbols for the channel decoder are exploited as a reference TS to refine the channel estimation. Here, the refined biased channel estimation rather than the unbiased estimation [28], [34] is considered due to the fact that the soft-decision SM symbols is unequal to the desired SM symbols. Then a more accurate channel estimate can be obtained by combining the refined channel estimate results with the TS-based channel estimate results. The details of the proposed decision-directed robust equalizers can be summarized as follows.

Step 1: Calculate the conditional first- and secondorder moment matrices based on the a priori MSE channel estimation results given in (7), where the a priori channel estimation results are given by the TS-based channel estimation results for its first iteration.

In the robust TDSDF-FDTE/FDSDF-FDTE, the estimated channel value and its error covariance matrix in (7) is directly employed to derive the optimal filter coefficients and the PDF of the equalized signal. According to [46], the conditional first- and second-order moment matrices of the real channel leaving an MSE channel estimation can also be applied to achieve more accurate CSI estimation than LS estimation, hence potentially resulting in a more robust receiver. Based on [46], and assuming that the channel matrix $\mathbf{H}$ represents a Rayleigh fading channel, the conditional first- and second-order moment matrices of the real channel $\mathbf{H}$ leaving an MSE estimate $\tilde{\mathbf{H}}$ is calculated as follows

$$
\begin{aligned}
\tilde{\mathbf{H}}_{p} & \triangleq E\left\{\mathbf{H}_{p} \mid \hat{\mathbf{H}}\right\}=E\left\{\mathbf{H}_{p} \hat{\mathbf{H}}_{p}^{H}\right\} E\left\{\hat{\mathbf{H}}_{p} \hat{\mathbf{H}}_{p}^{H}\right\}^{-1} \hat{\mathbf{H}}_{p} \\
& =\frac{\sigma_{h_{p}}^{2}}{\sigma_{h_{p}}^{2} \sigma^{2}}
\end{aligned}
$$
and

$$
\begin{aligned}
E\left\{\mathbf{H}_{p} \mathbf{H}_{p}^{H} \mid \hat{\mathbf{H}}\right\} & =E\left\{\mathbf{H}_{p} \mid \hat{\mathbf{H}}\right\} E\left\{\mathbf{H}_{p} \mid \hat{\mathbf{H}}\right\}^{H}+D\left\{\mathbf{H}_{p} \mid \hat{\mathbf{H}}\right\} \\
& =\tilde{\mathbf{H}}_{p} \tilde{\mathbf{H}}_{p}^{H}+D\left\{\mathbf{H}_{p} \mid \hat{\mathbf{H}}\right\},
\end{aligned}
$$

where

$$
\begin{aligned}
D\left\{\mathbf{H}_{p} \mid \hat{\mathbf{H}}\right\} & =E\left\{\mathbf{H}_{p} \mathbf{H}_{p}^{H}\right\}-E\left\{\mathbf{H}_{p} \hat{\mathbf{H}}_{p}^{H}\right\} E\left\{\hat{\mathbf{H}}_{p} \hat{\mathbf{H}}_{p}^{H}\right\}^{-1} E\left\{\hat{\mathbf{H}}_{p} \mathbf{H}_{p}^{H}\right\} \\
& =N_{t} \frac{\sigma_{h_{p}}^{2} \sigma_{\varepsilon_{p}}^{2}}{\sigma_{h_{p}}^{2}+\sigma_{\varepsilon_{p}}^{2}} \mathbf{I}_{N_{r}} .
\end{aligned}
$$

Therefore, the conditional first-order moment matrix $E\left\{\mathbf{D}_{k} \mid \hat{\mathbf{H}}\right\}$ and second-order moment matrix $E\left\{\mathbf{D}_{k} \mathbf{D}_{k}^{H} \mid \hat{\mathbf{H}}\right\}$ of the FD channel $\mathbf{D}_{k}$ can be reformulated as:

$$
\hat{\mathbf{D}}_{k} \triangleq E\left\{\mathbf{D}_{k} \mid \hat{\mathbf{H}}\right\}=\sum_{p=0}^{P-1} \tilde{\mathbf{H}}_{p} e^{-\frac{j 2 \pi p k}{K}},
$$




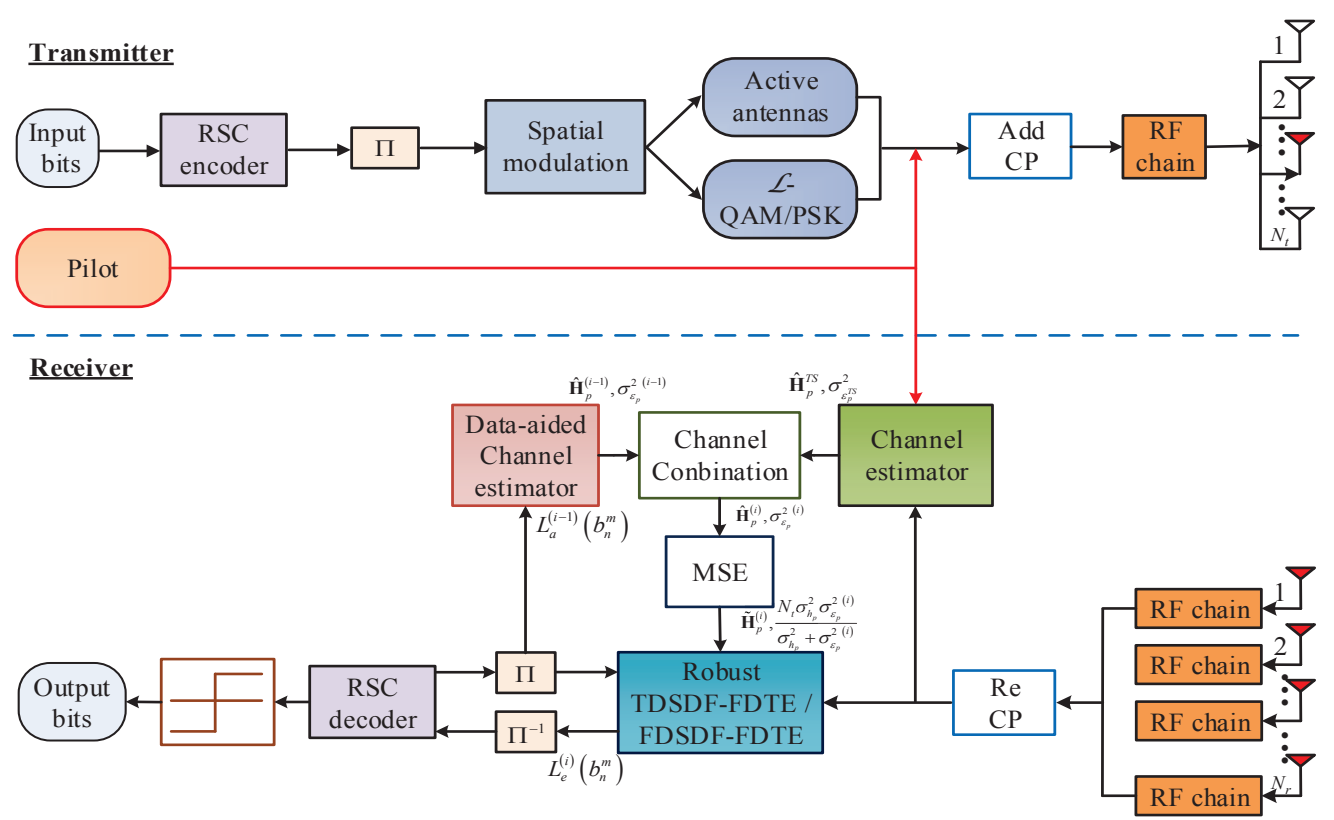

Fig. 3. Transceiver architecture of the proposed joint robust equalization, channel estimation and channel decoding aided SC-SM scheme with imperfect channel knowledge.

and

$$
E\left\{\mathbf{D}_{k} \mathbf{D}_{k}^{H} \mid \hat{\mathbf{H}}\right\}=\hat{\mathbf{D}}_{k} \hat{\mathbf{D}}_{k}^{H}+N_{t} \sum_{p=0}^{P-1} \frac{\sigma_{h_{p}}^{2} \sigma_{\varepsilon_{p}}^{2}}{\sigma_{h_{p}}^{2}+\sigma_{\varepsilon_{p}}^{2}} \mathbf{I}_{N_{r}}
$$

respectively.

Step 2: Perform the robust TDSDF-FDTE/FDSDFFDTE based on the conditional first- and second-order moment matrices of the real channel.

Upon replacing the real estimated first- and secondorder moment matrices (15)-(16) by the conditional firstand second-order moment matrices (47)-(48), the proposed robust TDSDF-FDTE/FDSDF-FDTE relying on the conditional first- and second-order moment matrices follows the same steps as that of the TDSDF-FDTE/FDSDFFDTE in the pervious subsections, except that the value of $\hat{\sigma}^{2}$ in (18) is refined to

$$
\hat{\sigma}^{2}=\operatorname{trace}\left(E\left\{\mathbf{x}_{k} \mathbf{x}_{k}^{H}\right\}\right) \sum_{p=0}^{P-1} \frac{\sigma_{h_{p}}^{2} \sigma_{\varepsilon_{p}}^{2}}{\sigma_{h_{p}}^{2}+\sigma_{\varepsilon_{p}}^{2}}+\sigma^{2} .
$$

Step 3: Invoke the soft-decision SM symbols output by the channel decoder as a reference TS to refine the channel estimation.

In contrast to the channel estimation model of Section II-B, by exploiting the soft sequence output by the channel decoder as a new TS, the received TD signal of the $r$ th RA can be expressed by

$\underbrace{\left[\begin{array}{c}y_{r, 0} \\ y_{r, 1} \\ \vdots \\ y_{r, K-1}\end{array}\right]}_{\mathbf{y}_{r} \in \mathbb{C}^{K \times 1}} \underbrace{\left[\begin{array}{cccc}\mathbf{s}_{0}^{T} & \mathbf{s}_{K-1}^{T} & \cdots & \mathbf{s}_{K-P+1}^{T} \\ \mathbf{s}_{1}^{T} & \mathbf{s}_{0}^{T} & \cdots & \mathbf{s}_{K-P+2}^{T} \\ \vdots & \vdots & \ddots & \vdots \\ \mathbf{s}_{K-1}^{T} & \mathbf{s}_{K-2}^{T} & \cdots & \mathbf{s}_{K-P}^{T}\end{array}\right]}_{\mathbf{S} \in \mathbb{C}^{K \times P N_{t}}} \underbrace{\left[\begin{array}{c}\mathbf{h}_{r}^{0} \\ \mathbf{h}_{r}^{1} \\ \vdots \\ \mathbf{h}_{r}^{P-1}\end{array}\right]}_{\mathbf{h}_{r} \in \mathbb{C}^{P N_{t} \times 1}}+\underbrace{\left[\begin{array}{c}n_{r, 0} \\ n_{r, 1} \\ \vdots \\ n_{r, K-1}\end{array}\right]}_{\mathbf{n}_{r} \in \mathbb{C}^{K \times 1}}$,

where $\mathbf{s}_{m} \in \mathbb{C}^{N_{t} \times 1}, m \in(0, K-1)$ is the desired SM symbol in the $m$ th interval defined in (2). Here, $\mathbf{h}_{r}^{p}=$ $\left[h_{1, r}^{p}, h_{2, r}^{p}, \cdots, h_{N_{t}, r}^{p}\right]^{T}$, and $h_{t, r}^{p}, p \in(0, P-1)$ stands for the element in the $r$ th-row and th-column of $\mathbf{H}_{p}$.
By entering the soft-decision SM output symbols into the channel decoder, according to the LS based channel estimation, the estimated channel $\hat{\mathbf{h}}_{r}$ can be formulated by

$$
\hat{\mathbf{h}}_{r}=\left(\overline{\mathbf{S}}^{H} \overline{\mathbf{S}}\right)^{-1} \overline{\mathbf{S}}^{H} \mathbf{y}_{r},
$$

where $\overline{\mathbf{S}} \triangleq E\{\mathbf{S}\}$. By substituting (50) into (51), we have

$$
\hat{\mathbf{h}}_{r}=\left(\overline{\mathbf{S}}^{H} \overline{\mathbf{S}}\right)^{-1} \overline{\mathbf{S}}^{H} \mathbf{S} \mathbf{h}_{r}+\left(\overline{\mathbf{S}}^{H} \overline{\mathbf{S}}\right)^{-1} \overline{\mathbf{S}}^{H} \mathbf{n}_{r}
$$

where $\hat{\mathbf{h}}_{r}$ is a biased estimate of $\mathbf{h}_{r}$ due to the fact that $\overline{\mathbf{S}} \neq$ $\mathbf{S}$, except at a high enough signal-to-noise ratio (SNR), where the bias becomes negligible. To tackle this issue, the biased channel estimation error covariance matrix can be calculated as

$$
\begin{aligned}
\boldsymbol{\Delta}_{\text {data }} \triangleq & E\left\{\left(\hat{\mathbf{h}}_{r}-\mathbf{h}_{r}\right)\left(\hat{\mathbf{h}}_{r}-\mathbf{h}_{r}\right)^{H}\right\} \\
= & E\left\{\left(\left(\overline{\mathbf{S}}^{H} \overline{\mathbf{S}}\right)^{-1} \overline{\mathbf{S}}^{H} \mathbf{S h}_{r}+\left(\overline{\mathbf{S}}^{H} \overline{\mathbf{S}}\right)^{-1} \overline{\mathbf{S}}^{H} \mathbf{n}_{r}-\mathbf{h}_{r}\right)\right. \\
& \left.\left(\left(\overline{\mathbf{S}}^{H} \overline{\mathbf{S}}\right)^{-1} \overline{\mathbf{S}}^{H} \mathbf{S} \mathbf{h}_{r}+\left(\overline{\mathbf{S}}^{H} \overline{\mathbf{S}}\right)^{-1} \overline{\mathbf{S}}^{H} \mathbf{n}_{r}-\mathbf{h}_{r}\right)^{H}\right\} \\
= & {\left[\left(\overline{\mathbf{S}}^{H} E\left\{\mathbf{S S}^{H}\right\}^{-1} \overline{\mathbf{S}}\right)^{-1}-\mathbf{I}_{P N_{t}}\right] E\left\{\mathbf{h}_{r} \mathbf{h}_{r}{ }^{H}\right\} } \\
+ & \sigma^{2}\left(\overline{\mathbf{S}}^{H} \overline{\mathbf{S}}\right)^{-1},
\end{aligned}
$$

where $E\left\{\mathbf{S S}^{H}\right\}$ is a diagonal second-order moment matrix due to the randomness of the transmitted SM symbol, given by

$$
E\left\{\mathbf{S S}^{H}\right\}=\operatorname{diag}\left[\sigma_{s_{0}}^{2}, \sigma_{s_{1}}^{2}, \cdots, \sigma_{s_{K-1}}^{2}\right],
$$

where

$$
\sigma_{s_{m}}^{2}=\operatorname{trace}\left(\sum_{p=0}^{P-1} E\left\{\mathbf{s}_{(m-p) \bmod K} \mathbf{s}_{(m-p) \bmod K}^{H}\right\}\right) .
$$

Moveover, $E\left\{\mathbf{h}_{r} \mathbf{h}_{r}{ }^{H}\right\}$ is the second-order moment matrix 
of the channel matrix in (2), computed as

$E\left\{\mathbf{h}_{r} \mathbf{h}_{r}{ }^{H}\right\}=\operatorname{diag}[\underbrace{\sigma_{h_{0}}^{2}, \cdots, \sigma_{h_{0}}^{2}}_{\mathbb{C}^{1 \times N_{t}}}, \cdots, \underbrace{\sigma_{h_{P-1}}^{2}, \cdots, \sigma_{h_{P-1}}^{2}}_{\mathbb{C}^{1 \times N_{t}}}]$

Similar to (10), due to the randomness of the transmitted SM symbols and the usage of the interleaver, the biased channel estimation error covariance matrix $\boldsymbol{\Delta}_{\text {data }}$ in (53) can also be approximated as a scaled diagonal matrix. Based on the channel estimation model in (7), $\boldsymbol{\Delta}_{\text {data }}$ can be expressed by

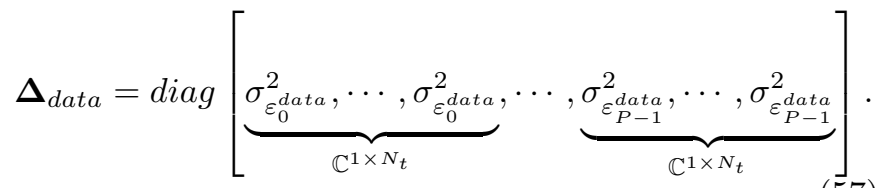

Therefore, we have

$$
\begin{aligned}
\sigma_{\varepsilon_{p}^{\text {data }}}^{2} & =\frac{1}{N_{t}} \sum_{i=1}^{N_{t}} a b s\left(\Delta_{p N_{t}+i, p N_{t}+i}^{\text {data }}\right), \\
\text { for } p & =0, \ldots, P-1,
\end{aligned}
$$

where $\Delta_{j, j}^{\text {data }}, j \in\left(0, P N_{t}\right)$ denotes the $j$ th diagonal element of $\boldsymbol{\Delta}_{\text {data }}$.

Next, the channel estimate based on the data sequence can be combined with the TS-based channel estimate, yielding the combined estimated channel $\hat{\mathbf{H}}_{p}^{c o m}$ with its error covariance $\sigma_{\varepsilon_{p}^{c o m}}^{2}$ being

$$
\hat{\mathbf{H}}_{p}^{\text {com }}=\sigma_{\varepsilon_{p}^{\text {com }}}^{2}\left(\frac{\hat{\mathbf{H}}_{p}^{\text {data }}}{\sigma_{\varepsilon_{p}^{\text {data }}}^{2}}+\frac{\hat{\mathbf{H}}_{p}^{T S}}{\sigma_{\varepsilon_{p}^{T S}}^{2}}\right),
$$

with

$$
\sigma_{\varepsilon_{p}^{c o m}}^{2}=\frac{1}{\frac{1}{\sigma_{\varepsilon_{p}^{d a t a}}^{2}}+\frac{1}{\sigma_{\varepsilon_{p}^{T S}}^{2}}} .
$$

Finally, the combined channel estimates will be employed as the a priori channel estimates for the next iteration. Note that although the proposed robust equalizers and the proposed decision-directed equalizers are designed by relying on the TD orthogonal training method, they can be readily extended to other estimation method, such as superimposed training [29] or FD pilot insertion [32].

Finally, the differences among the existing equalisers and the proposed equalisers are summarized in Table I to clarify the novelty of our works.

\section{Computational Complexity Analysis}

In this subsection, the computational complexities of both the proposed robust equalizers and of the decisiondirected equalizers are analyzed in terms of the number of real-valued multiplications. Specifically, for matrices $\mathbf{A} \in$ $\mathbb{C}^{m \times n}, \mathbf{B} \in \mathbb{C}^{n \times p}$, and $\mathbf{C} \in \mathbb{C}^{n \times 1}$, the operations of $\mathbf{A B}$ and $\|\mathbf{C}\|_{F}^{2}$ require $4 m n p$ and $2 n$ real-valued multiplication$\mathrm{s}$, respectively. The complexity order of the conventional equalizer is available in [19]. Furthermore, the complexities of both our proposed robust equalizers and of our decisiondirected equalizers are summarized in (61) in terms of the number of real-valued multiplications, where the 2based butterfly FFT and Inverse FFT (IFFT) operations are invoked in all the simulated equalizers. Finally, the complexity comparisons for specific system parameters are given in Section $\mathrm{V}$ to illustrate the calculations in (61).

\section{EXIT ChaRT ANALysis}

In this section, we present our EXIT chart analysis for characterizing the convergence behaviors of the proposed solutions along with the conventional DFE [19] in a twostage concatenated RSC-coded SC-SM relying on imperfect channel knowledge under different antenna configurations. In our simulations, the half-rate RSC $(2,1,2)$ code having an octal generator polynomial of $\left(G_{r}, G\right)=(7,5)_{8}$ is employed, and both the estimated channel having a fixed error variance and the real estimated channel presented in Section II-B are considered for demonstrating the superiority of both the proposed robust equalizers and of the decision-directed equalizers. Furthermore, a frequencyselective Rayleigh fading channel having a channel impulse response (CIR) length of $P=15$ is employed in our simulations, and the CIR taps are i.i.d. $\mathcal{C N}(0,1 / P)$ complex random variables.

\section{A. Convergence Behavior Analysis}

We consider block-based transmission of $K=256 \mathrm{SM}$ symbols having $(P-1) \mathrm{CP}$ length in each block. Based on the EXIT chart analysis [41], the convergence behaviors of the proposed equalizers are analysed for both full-rank and rank-deficient antenna configurations. Here, the curves labeled with 'T-FDTE' and 'F-FDTE' are the EXIT curves of the proposed robust TDSDF-FDTE and FDSDFFDTE, respectively. Furthermore, the curves labeled with 'd-T-FDTE' and 'd-F-FDTE' are the EXIT curves of the proposed decision-directed TDSDF-FDTE and decisiondirected FDSDF-FDTE, respectively.

Fig. 4 portrays the EXIT charts of both the conventional equalizer and of the proposed equalizers using $N_{t}=4$, $N_{r}=4$ and $N_{t}=8, N_{r}=4$ in the fixed-variance imperfect channel. Observe from Fig. 4 that all the proposed equalizers provide more extrinsic information than the conventional DFE in [19], which implies that the proposed FDTEs outperform the conventional DFE in terms of BER under both the full-rank and rank-deficient antenna configurations. It is also shown in Fig. 4 that all the proposed decision-directed equalizers offer better EXIT chart performances. This is due to the fact that by entering both the MSE estimate of the channel and the softdecision SM output symbols of the channel decoder into refining the channel estimation, the proposed decisiondirected equalizers attain a more accurate channel estimation, leading to a better BER performance. Moveover, the EXIT trajectories of the robust TDSDF-FDTE as well as the EXIT curve of the RSC $(2,1,2)$ code are plotted for visualizing the convergence behavior of the proposed equalizers, where the EXIT trajectories can reach their point of convergence after four iterations.

Next, Fig. 5 illustrates the EXIT charts of the real estimated channel by assuming a length $N=P$ ideal unit-power TS and the LS algorithm presented in Section II-B under both the full-rank and rank-deficient antenna configurations. As observed from Fig. 5, similar EXIT performances trends are demonstrated in Fig. 4. Furthermore, in Fig. 5, the proposed equalizers exhibit less superior behaviour than the conventional equalizer of [19] compared to those of the fixed-variance channel error estimation in Fig. 4, owing to the fact that the LS estimation can provide 
TABLE 1

COMPARISONS OF THE CONVENTIONAL EQUALISERS AND THE PROPOSED EQUALIZERS.

\begin{tabular}{|c|c|c|c|c|c|c|c|c|c|}
\hline & {$[35]-2014$} & {$[18]-2015$} & {$[36]-2016$} & {$[21]-2017$} & {$[19]-2017$} & {$[22]-2017$} & {$[20]-2018$} & [23]-2018 [Proposed \\
\hline \hline Soft-Input Soft-Output & $\checkmark$ & $\checkmark$ & $\checkmark$ & $\checkmark$ & $\checkmark$ & $\checkmark$ & $\checkmark$ & $\checkmark$ & $\checkmark$ \\
\hline Designed for SC-SM & & $\checkmark$ & & $\checkmark$ & $\checkmark$ & $\checkmark$ & $\checkmark$ & $\checkmark$ & $\checkmark$ \\
\hline $\begin{array}{c}\text { Priori LLRs } \\
\text { employed in Filter Design }\end{array}$ & $\checkmark$ & & $\checkmark$ & $\checkmark$ & $\checkmark$ & $\checkmark$ & $\checkmark$ & $\checkmark$ \\
\hline $\begin{array}{c}\text { Non-Gaussian Properties } \\
\text { of the Transmit Symbols }\end{array}$ & & & & & & & $\checkmark$ & $\checkmark$ & \\
\hline Feedback Structure & $\checkmark$ & & $\checkmark$ & & $\checkmark$ & & & $\checkmark$ \\
\hline Imperfect Feedback Decision & & & & & $\checkmark$ & & & $\checkmark$ \\
\hline $\begin{array}{c}\text { Imperfect Channel Estimation } \\
\text { Joint Channel Estimation } \\
\text { and Equalization }\end{array}$ & $\checkmark$ & & $\checkmark$ & & & $\checkmark$ & & $\checkmark$ \\
\hline Unbiased Channel Estimation & & & $\checkmark$ & & & & & $\checkmark$ \\
\hline
\end{tabular}

$$
\begin{aligned}
& C_{T D S D F-F D T E}=\underbrace{2 N_{r} K \log _{2} K}_{\text {FFT }}+\underbrace{2 N_{t} K \log _{2} K+4 N_{t} N_{r} K+4 N_{t}^{2} P K-4 N_{t}^{2} K}_{(4)}+\underbrace{K 2^{B+1}}_{(5)}+\underbrace{5 K 2^{B}}_{(19)} \\
& +\underbrace{4 N_{t}^{2} K}_{(20)}+\underbrace{14 N_{t}^{3} K+8 N_{t}^{2} N_{r} K+6 N_{t}^{2} K+N_{t}}_{(21)}+\underbrace{4 N_{t}^{2} N_{r} K+2 N_{t}^{2}}_{(25)}+\underbrace{8 N_{t}^{3}}_{(29)}+\underbrace{4 N_{t}^{2}}_{(32)} \\
& +\underbrace{4 N_{t}^{3} K+6 N_{t}^{3} P^{3}-18 N_{t}^{3} P^{2}+18 N_{t}^{3} P+4 N_{t}^{2} P^{2} K+6 N_{t}^{2} P^{2}-2 N_{t}^{2} K-12 N_{t}^{2} P+12 N_{t}^{2}+2 N_{t} P-2 N_{t}}_{(22)} \\
& +\underbrace{N_{t} K 2^{B+2}+B K 2^{B}+N_{t}^{2} 2^{B+2}}_{(31)}+\underbrace{4 N_{t}^{2} P K-4 N_{t}^{2} K}_{(13)}+\underbrace{16 N_{t}^{3} K+4 N_{t}^{3}}_{(30)}, \\
& C_{F D S D F-F D T E}=\underbrace{2 N_{r} K \log _{2} K}_{\mathrm{FFT}}+\underbrace{K 2^{B+1}}_{(5)}+\underbrace{2 N_{t} K \log _{2} K}_{(14)}+\underbrace{5 K 2^{B}}_{(19)}+\underbrace{4 N_{t}^{2} K}_{(20)}+\underbrace{16 N_{t}^{3} K+4 N_{t}^{3}}_{(30)}+\underbrace{4 N_{t}^{2}}_{(32)} \\
& +\underbrace{14 N_{t}^{3} K+8 N_{t}^{2} N_{r} K+6 N_{t}^{2} K+N_{t}}_{(36)}+\underbrace{10 N_{t}^{3} K+12 N_{t}^{3}+6 N_{t}^{2} K+16 N_{t}^{2}}_{(38)}+\underbrace{4 N_{t}^{2} N_{r} K+2 N_{t}^{2}}_{(41)} \\
& +\underbrace{N_{t} K 2^{B+2}+B K 2^{B}+N_{t}^{2} 2^{B+2}}_{(31)}+\underbrace{2 N_{t} K \log _{2} K+4 N_{t} N_{r} K+4 N_{t}^{2} K}_{(33)}+\underbrace{4 N_{t}^{3} K}_{(37)}+\underbrace{8 N_{t}^{3}}_{(43)}, \\
& C_{\text {data-aided equalizer }}=C_{T D S D F-F D T E} / C_{F D S D F-F D T E}+\underbrace{2 P^{3} N_{t}^{3}+8 P^{2} N_{t}^{2} K+6 P^{2} N_{t}^{2}+4 P N_{t} K}_{(51)} \\
& +\underbrace{2 P^{3} N_{t}^{3}+4 P^{2} N_{t}^{2} K+8 P^{2} N_{t}^{2}+2 P N_{t} K+K}_{(53)} .
\end{aligned}
$$
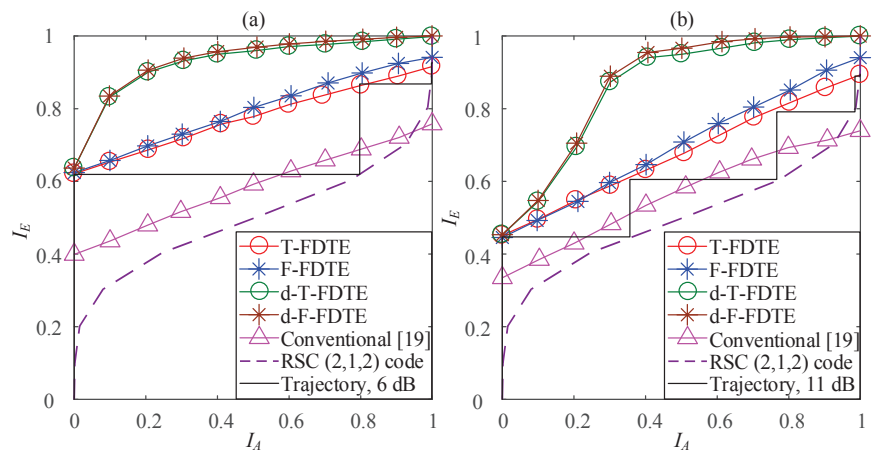

Fig. 4. EXIT chart comparisons between the conventional equalizer and the proposed robust equalizers in the fixed-variance imperfect channel employing QPSK, $P=15$, and $K=256$ with different antenna configurations and channel estimation errors: (a) $N_{t}=4$, $N_{r}=4, \sigma_{\varepsilon_{p}}^{2}=0.02 ;$ (b) $N_{t}=8, N_{r}=4, \sigma_{\varepsilon_{p}}^{2}=0.03$. Here, the EXIT trajectory of the proposed robust TDSDF-FDTE is calculated by assuming the interleaver length is 40,960 bits.

a more accurate estimation at a high SNR, leaving the channel error covariance based technique less competitive.

\section{B. Maximum Achievable Throughput}

According to the area property of EXIT charts [41], in this subsection, the maximum achievable throughput
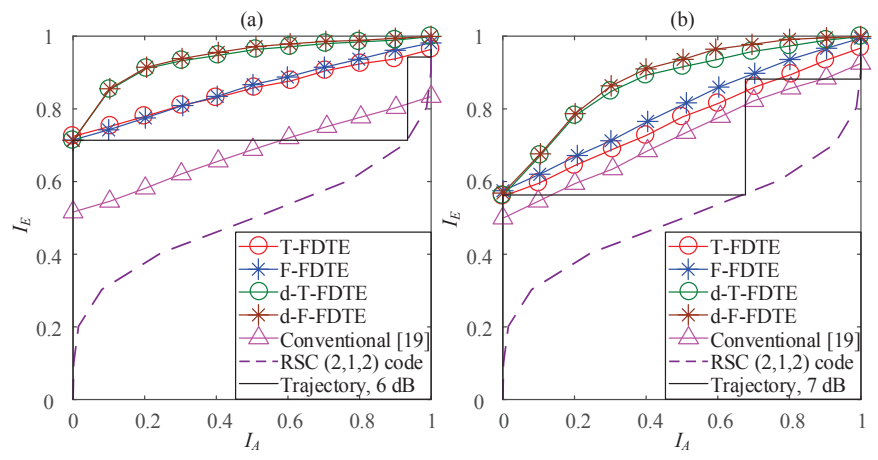

Fig. 5. EXIT chart comparisons between the conventional equalizer and the proposed robust equalizers in the real estimated channel employing QPSK, $N=P=15$, and $K=256$ with different antenna configurations: (a) $N_{t}=4, N_{r}=4$; (b) $N_{t}=8, N_{r}=4$. Here, the EXIT trajectory of the proposed robust TDSDF-FDTE is calculated by assuming the interleaver length is 40,960 bits.

of the proposed equalizers subjected to fixed-variance channel estimation error is characterized in Figs. 6 and 7 by assuming the same configurations as in Figs. 4 and 5 , respectively. The maximum achievable throughput of the conventional equalizer [19] is also added for both fullrank and rank-deficient TA-RA configurations. As shown in [18] and [22]-[23], it is challenging to derive the theoretical maximum throughput of suboptimal soft-decision 
detectors owing to the excessive complexity imposed by long CIRs. Consequently, the potent semi-analytical technique based on the area property of EXIT charts can be applied [41], which can be calculated as follows: $C_{\text {EXIT }}=$ $\mathcal{A}(\rho) R_{\mathrm{SM}}$ bits/symbol. Explicitly, $0 \leq \mathcal{A}(\rho) \leq 1$ refers to the area under the EXIT curve of the proposed equalizers at $\mathrm{SNR}=(\rho)$ and $R_{\mathrm{SM}}$ represents the bandwidth efficiency of the SC-SM system, which is quantified in bits per channel use (bpcu). To this end, the more extrinsic information the proposed equalizers provide, the higher $C_{\text {EXIT }}$ will become for a given $\mathrm{SNR}=(\rho)$. However, both the conventional equalizer of [19], as well as the proposed robust TDSDF-FDTE and FDSDF-FDTE may exhibit a BER error floor at a high SNR, so that the value of $\mathcal{A}(\rho)$ may not approach the $\left(I_{A}, I_{E}\right)=(1,1)$ point at the top right corner of the EXIT-chart, leading to a lower than the theoretical value of $C_{\text {ExIT }}$. This situation can be circumvented by exploiting the MSE estimation of the channel and the data sequence for aiding the channel decoder to refine the channel estimation in our decisiondirected equalizers.

As depicted in Figs. 6 and 7, when employing a half-rate RSC $(2,1,2)$ code for SC-SM systems, the system's spectral efficiency becomes $\frac{1}{2} R_{\mathrm{SM}}$, resulting in bpcu $=2$ and $2.5 \mathrm{bp}$ $\mathrm{s} / \mathrm{Hz}$ for the antenna configurations of $N_{t}=4, N_{r}=4$ and $N_{t}=8, N_{r}=4$, respectively (see horizontal dashed lines). For the case of the fixed-variance channel error of Fig. 6 , the max bpcu limits of the proposed robust TDSDFFDTEs and FDSDF-FDTEs schemes can be reached at $\rho=-0.3$ for $N_{t}=4, N_{r}=4, \sigma_{\varepsilon_{p}}^{2}=0.02$ in Fig. 6 (a) and at $\rho=3.1$ for $N_{t}=8, N_{r}=4, \sigma_{\varepsilon_{p}}^{2}=0.03$ in Fig. 6 (b). Correspondingly, the proposed decision-directed equalizers' bpcu values are reached at $\rho=-1.7$ for $N_{t}=4$, $N_{r}=4, \sigma_{\varepsilon_{p}}^{2}=0.02$ in Fig. 6 (a) and $\rho=0.7$ for $N_{t}=8$, $N_{r}=4, \sigma_{\varepsilon_{p}}^{2}=0.03$ in Fig. 6 (b). Moveover, for the realistically estimated channel of Fig. 7 , the bpcu limits of the proposed robust TDSDF-FDTEs and FDSDF-FDTEs are reached at $\rho=1.6$ for $N_{t}=4, N_{r}=4$ in Fig. 7 (a) and at $\rho=2.7$ for $N_{t}=8, N_{r}=4$ in Fig. 7 (b). Accordingly, the proposed decision-directed equalizers' bpcu values can be reached at $\rho=0.5$ for $N_{t}=4, N_{r}=4$ in Fig. 7 (a) and at $\rho=1.6$ for $N_{t}=8, N_{r}=4$ in Fig. 7 (b). As seen from Figs. 6 and 7, all the proposed FDTEs are capable of outperforming the conventional DFE design of [19] in dealing with the channel estimate error.

\section{Simulation Results}

In this section, we compare the proposed robust nonlinear FDTEs to the conventional DFE [19] along with the decision-directed equalizers in a half-rate RSC $(2,1,2)$ coded SC-SM scheme relying on imperfect channel knowledge. Both the BER performance v.s. the computational complexity of the proposed equalizers and of the conventional DFE [19] are compared under various antenna configurations. Furthermore, we also compare these equalizers in both fixed-variance channel error and the real estimated channel scenarios.

\section{A. Performance v.s. Complexity Comparisons for Fixed- Variance Channel Error}

Fig. 8 compares the BER performances of the proposed equalizers and of the conventional equalizer [19] for the
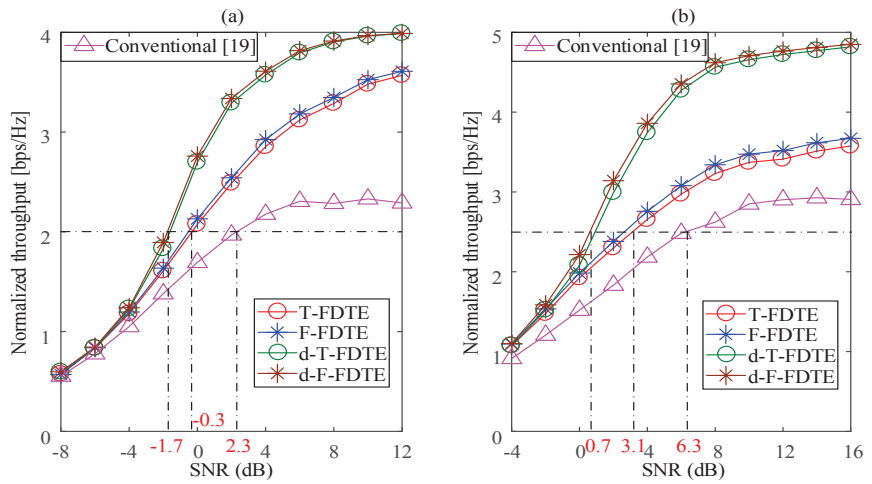

Fig. 6. Maximum achievable throughput of the conventional equalizer and the proposed robust equalizers in the fixed-variance imperfect channel employing QPSK, $P=15$, and $K=256$ with different antenna configurations and channel estimation errors: (a) $N_{t}=4$ $N_{r}=4, \sigma_{\varepsilon_{p}}^{2}=0.02$; (b) $N_{t}=8, N_{r}=4, \sigma_{\varepsilon_{p}}^{2}=0.03$. Here, the interleaver length adopted is 40,960 bits.
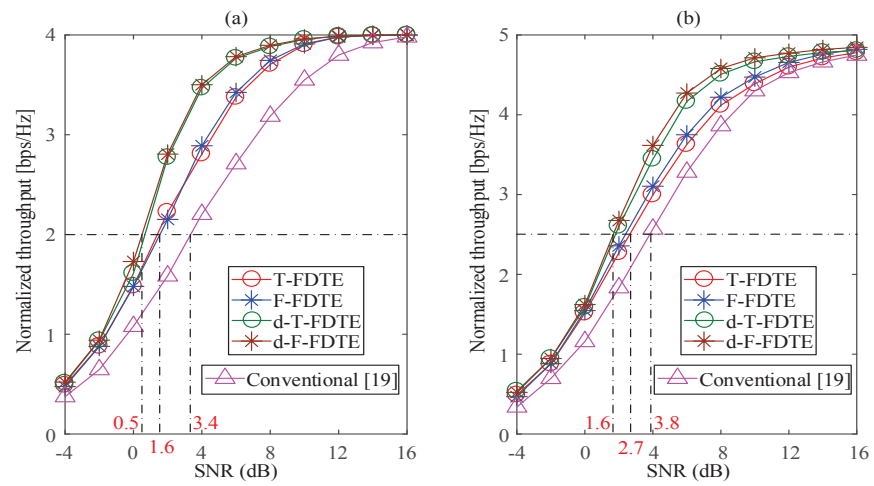

Fig. 7. Maximum achievable throughput of the conventional equalizer and the proposed robust equalizers in the real estimated channel employing QPSK, $N=P=15$, and $K=256$ with different antenna configurations: (a) $N_{t}=4, N_{r}=4$; (b) $N_{t}=8, N_{r}=4$. Here, the interleaver length adopted is 40,960 bits.

QPSK-modulated SC-SM system for both full-rank and rank-deficient antenna configurations using four turbo iterations. These curves are labeled in the same as Section IV. As shown in Fig. 8, all the proposed equalizers exhibit better BER performances than the conventional DFE of [19] in the case of imperfect channel knowledge. Moveover, the decision-directed equalizers, i.e., 'd-T-FDTE' and 'dF-FDTE' in Fig.8, exhibit similar performances, achieving the best BER performances in the simulations. This benefit is due to the fact that with the aid of the MSE estimate of the real channel and the data sequence, we iteratively refine the channel estimation. Given that the estimated channel becomes more accurate, we attain a gradually improved system performance, which is consistent with the EXIT chart predictions presented in Fig. 4.

Next, the complexities of the proposed robust equalizers are compared in Fig. 9 by assuming the same configuration as in Fig. 8. Compared to the conventional DFE of [19], the proposed robust TDSDF-FDTE provides considerable performance gains at the cost of about $22 \%$ increase in the complexity. Furthermore, the proposed robust FDSDFFDTE outperforms the conventional DFE of [19] by more than $4 \mathrm{~dB}$ at $\mathrm{BER}=10^{-2}$, despite $70 \%$ reduction in complexity. Finally, the decision-directed equalizers attain the best BER at the highest complexity. In summary, the proposed equalizers are capable of striking an attractive 

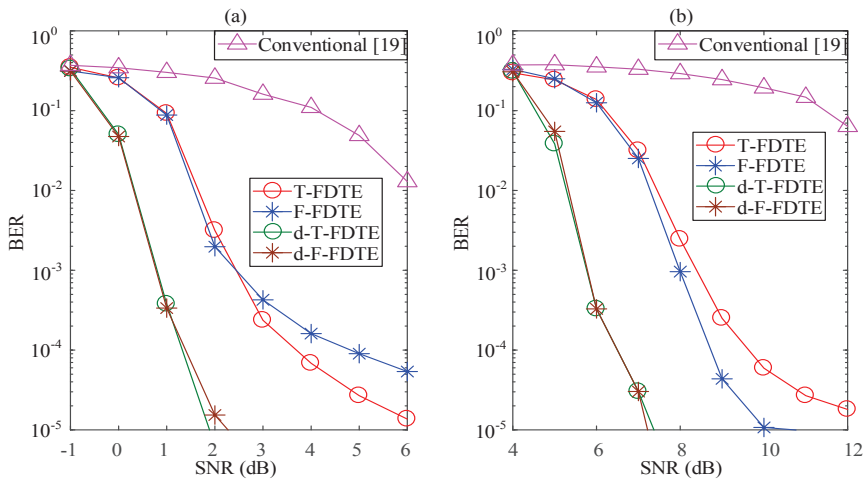

Fig. 8. BER performance comparison between the conventional equalizer and the proposed robust equalizers in the fixed-variance imperfect channel employing QPSK, $P=15$, and $K=256$ with different antenna configurations and channel estimation errors: (a) $N_{t}=4, N_{r}=4, \sigma_{\varepsilon_{p}}^{2}=0.02$; (b) $N_{t}=8, N_{r}=4, \sigma_{\varepsilon_{p}}^{2}=0.03$. Here the interleaver length adopted is 40960 bits.
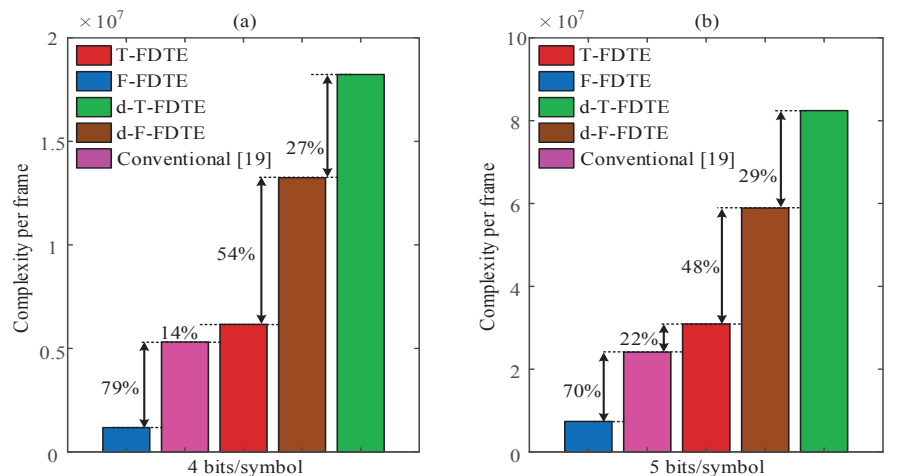

Fig. 9. Complexity comparison between the conventional equalizer and the proposed robust equalizers employing QPSK, $P=15$, and $K=256$ with different antenna configurations: (a) $N_{t}=4, N_{r}=4$; (b) $N_{t}=8, N_{r}=4$.

BER v.s. complexity trade-off both in full-rank and rankdeficient antenna scenarios.

Finally, Fig. 10 plots the BERs of the proposed robust equalizers for high channel estimation error variances of $\sigma_{\varepsilon_{p}}^{2}=0.08$ and $\sigma_{\varepsilon_{p}}^{2}=0.05$ using four iterations, where the channel variance $\sigma_{h_{p}}^{2}$ in the simulation is set to $1 / P \approx 0.067$. Observe that the proposed robust equalizers still outperform the conventional DFE of [19]. Moveover, the decision-directed equalizers still outperform the common robust equalizers at high channel estimation error variances. Therefore, our approach exploiting the MSE estimate of the channel and the soft-decision SM symbols for refining the channel estimation improves the accuracy of channel estimation, hence leading to beneficial BER gains compared to the common robust equalizers.

Fig. 11 shows the BER performance of the proposed equalizers both as a function of the SNR v.s. the channel estimation variance $\sigma_{\varepsilon_{p}}^{2}$ and v.s. the CIR length $P$ for four iterations. Observe from Fig. 11 (b) that the proposed robust equalizers achieve substantial BER performance improvements at diverse channel estimation variance levels over the conventional DFE of [19], especially for $\sigma_{\varepsilon_{p}}^{2} \geq$ 0.01. Furthermore, as seen from Fig. 11 (c), the proposed equalizers provide considerable BER gains at different CIR length over the conventional DFE of [19].

In Fig. 12, the performance curve of the MAP-based
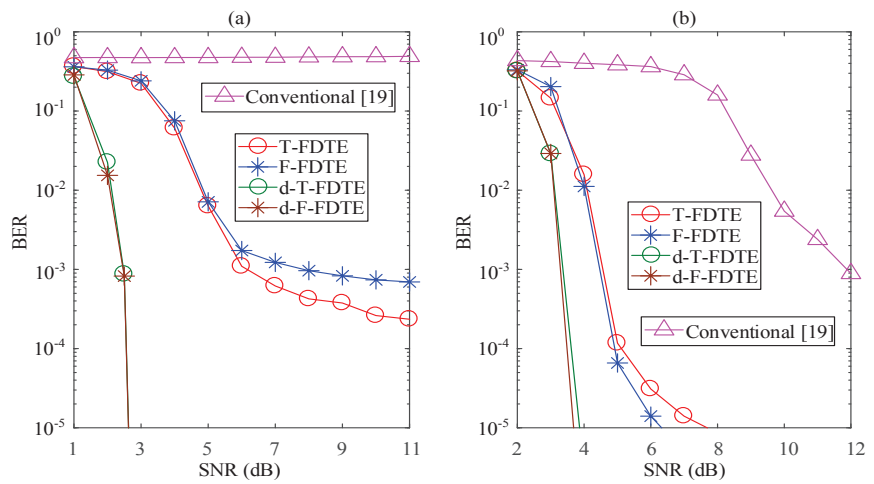

Fig. 10. BER performance comparison between the conventional equalizer and the proposed robust equalizers in the fixed-variance imperfect channel employing QPSK, $P=15$, and $K=256$ with different antenna configurations and channel estimation errors: (a) $N_{t}=8, N_{r}=8, \sigma_{\varepsilon_{p}}^{2}=0.08$ with an interleaver having 40,960 bits; (b) $N_{t}=16, N_{r}=8, \sigma_{\varepsilon_{p}}^{2}=0.05$ with an interleaver having 38,400 bits.
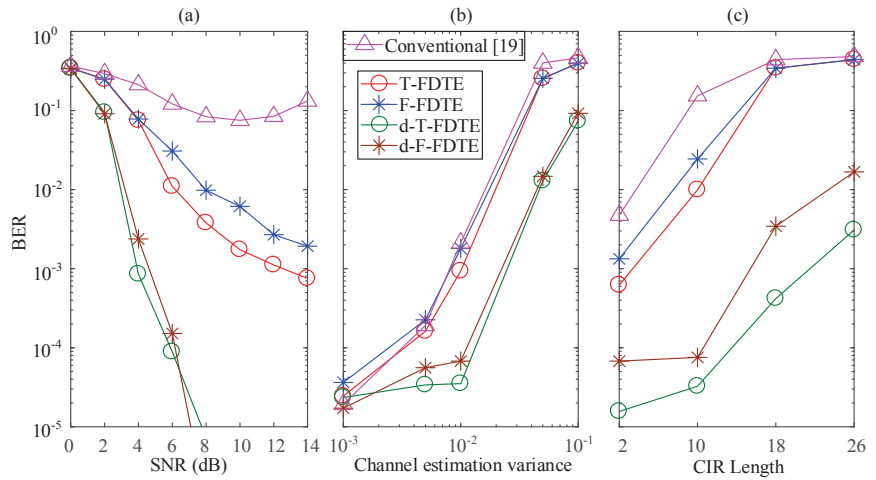

Fig. 11. BER v.s. SNR, channel estimation variance and CIR length for the conventional equalizer and the proposed robust equalizers in the $2 \times 2$ SC-SM systems employing QPSK, $P=15$, and $K=256$ (a)BER v.s. SNR when $\sigma_{\varepsilon_{p}}^{2}=0.02$; (b) BER v.s. channel estimation variance when $\mathrm{SNR}=7 \mathrm{~dB}$; (c) $\mathrm{BER}$ v.s. CIR length at $\mathrm{SNR}=7 \mathrm{~dB}$, $\sigma_{\varepsilon_{p}}^{2}=1 /(5 P)$.

equalizer [22] relying on perfect CSI is added to show the performance gap between the proposed robust equalizers and the MAP-based equalizer [22] both in terms of its EXIT chart, its throughput and its BER performance for a low-complexity configuration of $N_{t}=2, N_{r}=2$, BPSK and $P=3$. As observed from Fig.12, there is still considerable performance gap between the MAP-based equalizer [22] and the proposed robust equalizers, although the latter can provide significant improvements over the conventional equalizer. Due to its high complexity, the MAP-based equalizer [22] having perfect CSI is unsuitable for high-dimensional configurations.

\section{B. Performance Comparisons in Real Estimated Channels}

Fig. 13 portrays the BER v.s. SNR performance for our different equalizers using four iterations for frequencyselective Rayleigh fading channels having a CIR length of $P=15$, where the $\mathrm{CIR}$ taps are i.i.d. $\mathcal{C N}(0,1 / P)$ complex random variables. In Fig. 13 (a) the normalized mean-square error (NMSE) is employed as our channel estimation quality metric for different TS lengths, which 

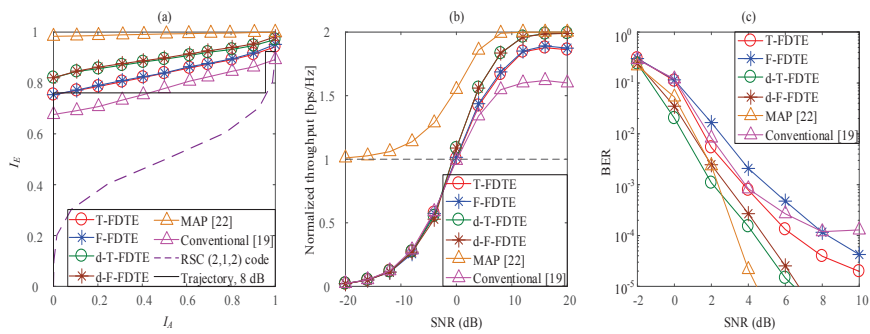

Fig. 12. EXIT chart, achievable throughput and BER performance comparisons between the conventional equalizer and the proposed robust equalizers in the fixed-variance imperfect channel employing BPSK, $N_{t}=2, N_{r}=2, \sigma_{\varepsilon_{p}}^{2}=0.05, P=3$, and $K=512$ with an interleaver having 40,960 bits: (a) EXIT chart comparison; (b) maximum achievable throughput; (c) BER performance comparison.
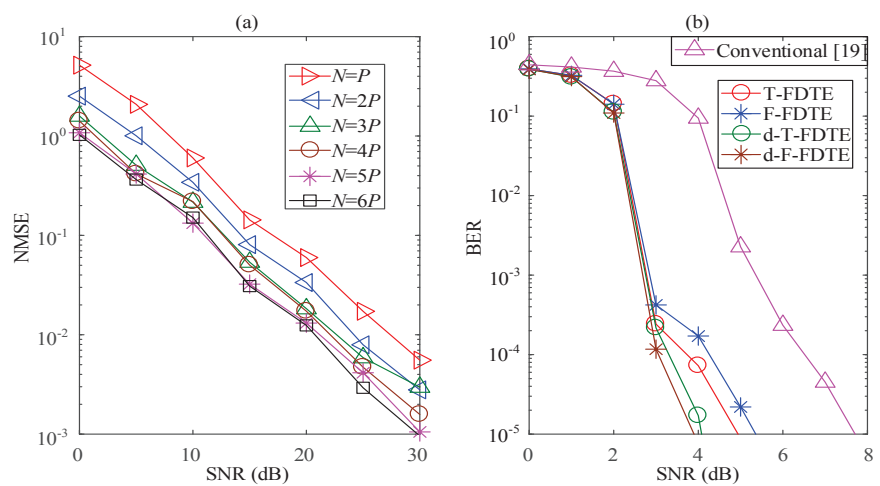

Fig. 13. Performance comparison between the conventional equalizer and the proposed equalizers for real estimated channels employing QPSK, $N_{t}=4, N_{r}=4, N=P=15$ and $K=256$ with an interleaver having 40,960 bits: (a) NMSE of the channel estimator v.s. SNR for different TS lengths; (b) BER v.s. SNR for different equalizers.

is defined as

$$
\begin{aligned}
& \operatorname{NMSE}=E\left(\frac{\left|\hat{h}_{t, r}^{p}-h_{t, r}^{p}\right|^{2}}{\left|h_{t, r}^{p}\right|^{2}}\right), \\
& t \in\left(1, N_{t}\right), r \in\left(1, N_{r}\right), p \in(0, P-1) .
\end{aligned}
$$

Next, in Fig. 13 (b), $N=P=15$ ideal unit-power TS [42] and the TD-based LS algorithm of Section II-B are applied to demonstrate the superiority of the proposed equalizers over the conventional equalizer of [19]. As seen from Fig. 13, the proposed robust TDSDF-FDTE and FDSDF-FDTE offer about $2.5 \mathrm{~dB}$ and $2 \mathrm{~dB}$ improvements over the conventional DFE of [19] for $N_{t}=4, N_{r}=4$ at the BER of $10^{-5}$, respectively. As shown in Fig. 13 (b), the decision-directed equalizers effectively alleviate the error floor of the common robust equalizers, hence leading to an improved BER performance.

Fig. 14 evaluates the BERs of the proposed equalizers for transmission over the Extended Vehicular A (EVA) channel model using four iterations. Specifically, the system's bandwidth is set to $3.84 \mathrm{MHz}$ at a microwave carrier frequency of $2 \mathrm{GHz}$. The power delay profile of the fading channel is taken from [47] with the maximum Doppler frequency set to $222 \mathrm{~Hz}$ corresponding to a typical velocity of $120 \mathrm{~km} / \mathrm{h}$. The TS length is set to $N=P$. As illustrated in Fig. 14, the proposed robust equalizers still perform better than the conventional DFE of [19] in the time-varying channel with the decision-directed equalizers achieving the best BER performances, which is consistent with the trends presented above.
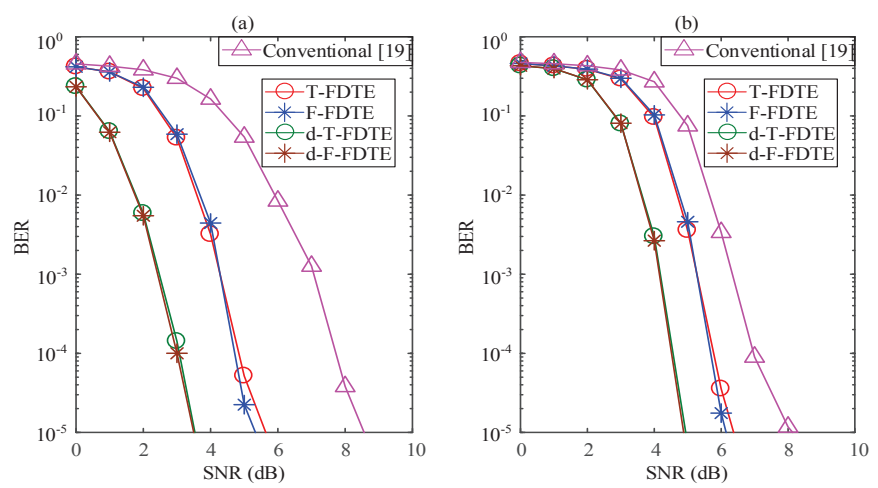

Fig. 14. BER performance comparison between the conventional equalizer and the proposed robust equalizers in the estimated EVA channel employing QPSK, $K=256$, and $N=P$ with different antenna configurations: (a) $N_{t}=4, N_{r}=4$ with an interleaver having 1,024 bits; (b) $N_{t}=8, N_{r}=4$ with an interleaver having 1,280 bits.

\section{Conclusions}

This paper proposed several robust DFE designs for our CP-aided SC-SM system relying on realistic imperfect channel knowledge. Compared to the conventional DFE design based on the assumption of idealized perfect channel estimation [19], the proposed robust designs overcomes the degradations imposed by the channel estimation error, making it eminently suitable for practical wireless systems. Moveover, both the optimal coefficients involved in the robust designs and the decision-directed joint design conceived with channel estimation are addressed in this paper. Finally, biased channel estimation is also considered in our decision-directed joint design conceived with channel estimation, rather than simply assuming idealized unbiased estimation [28], [34]. Both our EXIT chart analysis and simulation results show that the proposed robust equalizers provide considerable performance gains over the conventional DFE of [19] in the presence of realistic imperfect channel knowledge.

\section{REFERENCES}

[1] M. Di Renzo, H. Haas, A. Ghrayeb, S. Sugiura, and L. Hanzo, "Spatial modulation for generalized MIMO: challenges, opportunities and implementation," Proc. IEEE, vol. 102, no. 1, pp. 56-103, Jan. 2014.

[2] M. Di Renzo, H. Haas, and P. Grant, "Spatial modulation for multiple-antenna wireless systems: A survey," IEEE Commun. Mag., vol. 49, no. 12, pp. 182-191, Dec. 2011.

[3] R. Y. Mesleh, H. Haas, S. Sinanovic, C. W. Ahn, and S. Yun, "Spatial modulation," IEEE Trans. Veh. Technol., vol. 57, no 4, pp. 2228-2241, Jul. 2008.

[4] S. Sugiura, S. Chen, and L. Hanzo, "A universal space-time architecture for multiple-antenna aided systems," IEEE Commun. Surveys Tuts., vol. 14, no. 2, pp. 401-420, May 2012.

[5] T. L. Narasimhan, P. Raviteja, and A. Chockalingam, "Generalized spatial modulation in large-scale multiuser MIMO systems," IEEE Trans. Wireless Commun., vol. 14, no. 7, pp. 37643779, Jul. 2015.

[6] N. Serafimovski, R. Mesleh, P. Chambers, M. Di Renzo, C. Wang, P. Grant, M. Beach, and H. Haas, "Practical implementation of spatial modulation," IEEE Trans. Veh. Technol., vol. 62 , no. 9, pp. 4511-4523, Nov. 2013.

[7] W. Wang and W. Zhang, "Transmit signal designs for spatial modulation with analog phase shifters," IEEE Trans. Wireless Commun., vol. 17, no. 5, pp. 3059-3070, May 2018.

[8] R. Rajashekar, K. Hari, and L. Hanzo, "Quantifying the transmit diversity order of Euclidean distance based antenna selection in spatial modulation," IEEE Sig. Process. Lett., vol. 22, no. 9, pp. 1434-1437, Sep. 2015. 
[9] S. Guo, H. Zhang, P. Zhang, D. Wu, and D. Yuan, "Generalized 3-D constellation design for spatial modulation," IEEE Trans. Commun., vol. 65, no. 8, pp. 3316-3327, Aug. 2017.

[10] P. Yang, Y. Xiao, Y. Guan, K. Hari, A. Chockalingam, S. Sugiura, H. Haas, M. Di Renzo, C. Masouros, Z. Liu, L. Xiao, S. Li, and L. Hanzo, "Single-carrier spatial modulation: A promising design for large-scale broadband antenna systems," IEEE Commun. Surveys Tuts., vol. 18, no. 3, pp. 1687-1716, Feb. 2016.

[11] B. Gong, L. Gui, Q. Qin, and X. Ren, "Compressive sensingbased detector design for SM-OFDM massive MIMO high speed train systems," IEEE Trans. Broadcast., vol. 63, no. 4, pp. 714726, Dec. 2017

[12] H. Ngo, C. Xu, S. Sugiura, and L. Hanzo, "Space-time-frequency shift keying for dispersive channels," IEEE Signal Proc. Lett. vol. 18, no. 3, pp. 177-180, Mar. 2011

[13] P. Som and A. Chockalingam, "Spatial modulation and space shift keying in single carrier communication," in Proc. IEEE PIMRC, Sydney, Australia, Sep. 2012, pp. 1991-1996.

[14] R. Rajasherkar, K. Hari, and L. Hanzo, "Spatial modulation aided zero-padded single carrier transmission for dispersive channels," IEEE Trans. Commun., vol. 61, no. 6, pp. 2318 2329, Jun. 2013.

[15] S. Wang, Y. Li, and J. Wang, "Multiuser detection in massive spatial modulation MIMO with low-resolution ADCs," IEEE Trans. Wireless Commun., vol. 14, no. 4, pp. 2156-2168, Apr. 2015.

[16] A. Glavieux, C. Laot, and J. Labat, "Turbo equalization over a frequency selective channel," in Proc. Int. Symp. Turbo Codes, Brest, France, Sep. 1997, pp. 96-102.

[17] C. Douillard, M. Jézéquel, C. Berrou, A. Picart, P. Didier, and A. Glavieux, "Iterative correction of intersymbol interference: Turbo equalization," Eur. Trans. Telecommun., vol. 6, pp. 507511, Sept.-Oct. 1995.

[18] S. Sugiura and L. Hanzo, "Single-RF spatial modulation requires single-carrier transmission: Frequency-domain turbo equalization for dispersive channels," IEEE Trans. Veh. Technol., vol. 64, no. 10 , pp. $4870-4875$, Oct. 2015

[19] Y. Zhao, P. Yang, Y. Xiao, et al. "An improved frequency domain turbo equalizer for single-carrier spatial modulation systems," IEEE Trans. Veh. Technol., vol. 66, no. 8, pp. 75687572, Aug. 2017.

[20] Y. Zhao, Y. Xiao, et al. "Generalized approximate message passing aided frequency domain turbo equalizer for single-carrier spatial modulation," IEEE Trans. Veh. Technol., vol. 67, no. 4, pp. 3630-3634, Apr. 2018.

21] L. He, J. Wang, and J. Song, "A priori information aided iterative equalization: a novel approach for single-carrier spatial modulation in dispersive channels," IEEE Trans. Veh. Technol., vol. 66 , no. 5, pp. 4448-4452, May 2017.

[22] L. Xiao, Y. Xiao, Y. Zhao, et al. "Time-domain turbo equalization for single-carrier generalized spatial modulation systems," IEEE Trans. Wireless Commun., vol. 16, no. 9, pp. 5702-5716, Sep. 2017.

[23] Y. Zhao, P. Yang, Y. Xiao, et al. "Soft-feedback time-domain turbo equalization for single-carrier generalized spatial modulation," IEEE Trans. Veh. Technol., vol. 67, no. 10, pp. 9421-9434, Oct. 2018.

[24] N. Benvenuto, R. Dinis, D. Falconer, and S. Tomasin, "Single carrier modulation with nonlinear frequency domain equalization: An idea whose time has come-again," Proc. IEEE, vol. 98, no. 1, pp. 69-96, Jan. 2010.

[25] M. Di Renzo and H. Haas, "Space shift keying (SSK) modulation with partial channel state information: Optimal detector and performance analysis over fading channels," IEEE Trans. Commun., vol. 58, no. 11, pp. 3196-3210, Nov. 2010

[26] E. Basar, U. Aygolu, E. Panayirci, and H. V. Poor, "Performance of spatial modulation in the presence of channel estimation errors," IEEE Commun. Lett., vol. 16, no. 2, pp. 176-179, Feb. 2012.

[27] M. Di Renzo, D. De Leonardis, F. Graziosi, and H. Haas, "Space shift keying (SSK-) MIMO with practical channel estimates" IEEE Trans. Commun., vol. 60, no. 4, pp. 998-1012, Apr. 2012.

[28] B. Ng, C. Lam, and D. Falconer, "Turbo frequency domain equalization for single-carrier broadband wireless systems," IEEE Trans. Wireless Commun., vol. 6, no. 2, pp. 759-767, Feb. 2007.

$[29]$ Q. Guo and D. Huang, "A frequency domain state-space approach to LS estimation and its application in turbo equalization," IEEE Trans. Signal Process., vol. 59, no. 7, pp. 3288-3300, Jul. 2011

[30] Z. Xie, X. Chen and X. Liu, "A virtual pilot-assisted channel estimation algorithm for MIMO-SCFDE systems over fast time- varying multipath channels," IEEE Trans. Veh. Technol., vol. 67, no. 6, pp. 4901-4909, Jul. 2018.

31] Y. R. Zheng and C. Xiao, "Channel estimation for frequencydomain equalization of single-carrier broadband wireless communications," IEEE Trans. Veh. Technol., vol. 58, no. 2, pp. 815-823, Feb. 2009.

[32] B. Zheng, F. Chen, Q. Guan, M. Wen, H. Yu, and F. Ji, "Novel pilot design and signal detection for SC-FDE systems with frequency-domain pilot multiplexing technique," IEEE Commun. Lett., vol. 19, no. 8, pp. 1466-1469, Aug. 2015.

[33] C. Lin, J. Wu, and T. Lee, "Robust receiver design for MIMO single-carrier block transmission over time-varying dispersive channels against imperfect channel knowledge," IEEE Trans. Wireless Commun., vol. 7, no. 10, pp. 3799-3812, Oct. 2008.

[34] Y. Zhu, P. Zhe, H. Zhou, and D. Huang, "Robust single carrier frequency domain equalization with imperfect channel knowledge," IEEE Trans. Wireless Commun., vol. 15, no. 9, pp. 60916103, Sep. 2016

[35] N. Souto, R. Dinis, and J. C. Silva, "Impact of channel estimation errors on SC-FDE systems," IEEE Trans. Commun., vol. 62 , no. 5 , pp. $1530-1540$, May 2014

[36] M. A. Khalighi, J. J. Boutros, and J. F. Helard, "Data-aided channel estimation for turbo-PIC MIMO detectors," IEEE Commun. Lett., vol. 10, no. 5, pp. 350-352, May 2006.

[37] B. Ning, R. Visoz, A.O. Berthet, "Semi-analytical performance prediction method for iterative MMSE-IC detection and semiblind channel estimation," in Proc. Vehic. Tech. Conf. (VTC) Spring, Hungrary, Budapest, May 2011, pp. 1-5.

[38] N. Benvenuto and S. Tomasin, "On the comparison between OFDM and single carrier modulation with a DFE using a frequency-domain feedforward filter," IEEE Trans. Commun. vol. 50, pp. 947-955, Jun. 2002

[39] M. Tüchler and J. Hagenauer, "Linear time and frequency domain turbo equalization," in Proc. Vehic. Tech. Conf. (VTC) Fall, Atlantic City, NJ, Oct. 2001, pp. 1449-1453.

[40] Z. Chen, Y. R. Zheng, J. Tao, J. Wang, and J. Song, "Frequency domain turbo equalization under MMSE criterion for single carrier MIMO systems," in Proc. IEEE GLOBECOM, San Diego, CA, USA, Dec. 2015, pp. 1-5.

[41] M. Hajjar and L. Hanzo, "EXIT charts for system design and analysis," IEEE Commun. Surveys Tuts., vol. 16, no. 1, pp. 127153, Feb. 2014.

[42] D. Chu, "Polyphase codes with good periodic correlation properties," IEEE Trans. Inform. Theory, vol. 18, no. 4, pp. 531-532, Jul. 1972.

[43] S. Sugiura, S. Chen, and L. Hanzo, "Coherent and differential space-time shift keying: A dispersion matrix approach," IEEE Trans. Commun., vol. 58, no. 11, pp. 3219-3230, Nov. 2010.

[44] N. Benvenuto and S. Tomasin, "Iterative design and detection of a DFE in the frequency domain," IEEE Trans. Commun., vol. 53 , no. 11 , pp. 1867-1875, Nov. 2005

[45] W. Han, Q. Yin, L. Bai, and B. Yao, "Joint transceiver design for iterative FDE," IEEE Trans. Signal Process., vol. 61, no. 13, pp. 3389-3405, Jul. 2013.

[46] A. P. Liavas, "Tomlinson-Harashima precoding with partial channel knowledge," IEEE Trans. Commun., vol. 53, no. 1, pp. 5-9, Jan. 2005.

[47] 3rd Generation Partnership Project, "Technical Specification Group Radio Access Network; evolved Universal Terrestrial Radio Access(UTRA): Base Station (BS) radio transmission and reception", TS 36.104, V8.7.0, Sep. 2009.

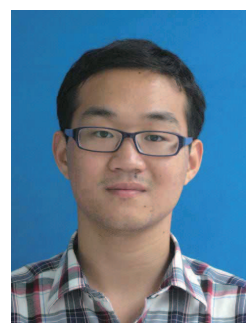

Yan Zhao received the B.E. degrees in communication engineering from Zhengzhou University, Zhengzhou, China, in 2012. Since Sep. 2013, he is working towards the Ph.D. degree in National Key Laboratory of Science and Technology on Communications in University of Electronic Science and Technology of China (UESTC). From 2017 to 2018, he was a Visiting Student with the School of Electronics and Computer Science, University of Southampton, U.K. Now his research interests include MIMO systems, non-orthogonal multiple access and communication signal processing. 


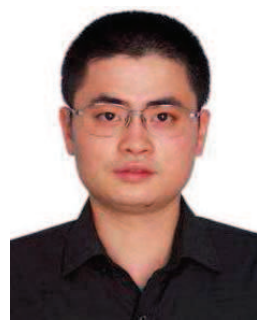

tions.

Yue Xiao (M'04) received the Ph.D. degree in communication and information systems from the University of Electronic Science and Technology of China in 2007. He is currently a Full Professor with the University of Electronic Science and Technology of China. He has authored over 30 international journals and involved in several projects in the Chinese Beyond 3G Communication Research and Development Program. His research interests are in the area of wireless and mobile communica-

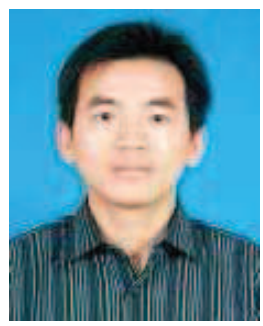

Ping Yang (M'13-SM'16) received the Ph.D. degree from the University of Electronic Science and Technology of China (UESTC) in 2013. From 2012 to 2013, he was a Visiting Student with the School of Electronics and Computer Science, University of Southampton, U.K. Since 2014, he has been a Research Fellow in EEE with NTU, Singapore. He is also an Associate Professor with UESTC. His current research interests include MIMO/OFDM, machine learning, life science, and communica-

tion signal processing.

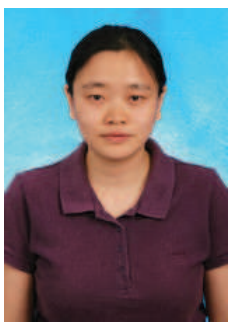

Binhong Dong received her Ph.D. in the Department of Communication and Information Engineering from University of Electronic Science and Technology of China in 2010. Her major research interests are mobile communication, anti-jamming communication, low intercept communication,etc.

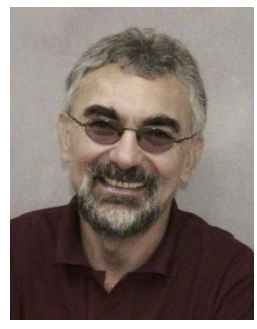

Lajos Hanzo (http://www-

mobile.ecs.soton.ac.uk) FREng, F'04, FIET, Fellow of EURASIP, received his 5-year degree in electronics in 1976 and his doctorate in 1983 from the Technical University of Budapest. In 2009 he was awarded an honorary doctorate by the Technical University of Budapest and in 2015 by the University of Edinburgh. In 2016 he was admitted to the Hungarian Academy of Science. During his 40-year career in telecommunications he has held various research and academic posts in Hungary, Germany and the UK. Since 1986 he has been with the School of Electronics and Computer Science, University of Southampton, UK, where he holds the chair in telecommunications. He has successfully supervised $119 \mathrm{PhD}$ students, co-authored 18 John Wiley/IEEE Press books on mobile radio communications totalling in excess of 10000 pages, published $1800+$ research contributions at IEEE Xplore, acted both as TPC and General Chair of IEEE conferences, presented keynote lectures and has been awarded a number of distinctions. Currently he is directing a 60-strong academic research team, working on a range of research projects in the field of wireless multimedia communications sponsored by industry, the Engineering and Physical Sciences Research Council (EPSRC) UK, the European Research Council's Advanced Fellow Grant and the Royal Society's Wolfson Research Merit Award. He is an enthusiastic supporter of industrial and academic liaison and he offers a range of industrial courses. He is also a Governor of the IEEE ComSoc and VTS. He is a former Editor-in-Chief of the IEEE Press and a former Chaired Professor also at Tsinghua University, Beijing. For further information on research in progress and associated publications please refer to http://www-mobile.ecs.soton.ac.uk 